\title{
Review Article \\ Death Anxiety in the Elderly in Iran: A Systematic Review and Meta-analysis
}

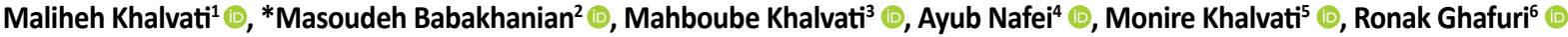

1. Department of Social Work, Faculty of Paramedical, Mashhad University of Medical Sciences, Mashhad, Iran.

2. Social Determinants of Health Research Center, Semnan University of Medical Sciences, Semnan, Iran.

3. Department of English Literature, Faculty of Human Sciences, University of South Africa, Pretoria, South Africa.

4. Department of Social Welfare Management, University of Social Welfare and Rehabilitation Science (USWR), Tehran, Iran.

5. Department of French Literature, Faculty of Foreign Languages, Central Tehran Branch, Islamic Azad University, Tehran, Iran.

6. Education Organization of District 9, Tehran, Iran.

$\begin{gathered}\text { Use your device to scan } \\ \text { and read the article online }\end{gathered}$
dolichtion: Kttps://doi.org/10.32598/sija.16.2.862.2

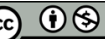

Received: 25 May 2020 Accepted: 02 Nov 2020 Available Online: $01 \mathrm{Jul} 2021$

Key words:

Elderly, Death anxiety, Systematic review, Meta-analysis

\section{A B S TR ACT}

Objectives Aging is one of the most critical stages of human development that has its own characteristics and conditions. One of the most common issues in old age is the mental health whose achievement requires special attention from both health system policymakers and service providers to the elderly. Death anxiety is one of the most common mental health issues in old age, because this period is full of feelings of shortcomings and disabilities. Since death anxiety is a multidimensional factor, it is expected to affect many aspects of the elderly. The present study aims to review and analyze published studies in the field of death anxiety in the elderly in Iran.

Methods \& Materials This is a systematic review and meta-analysis conducted on the studies related to the death anxiety of the elderly in Iran published in Persian from 2011 to 2019. A search was conducted in national databases, including SID, IranDoc, MagIran, IDML, and CIVILICA using the keywords: Death anxiety, elderly, and older adult. Initial search yielded 61 articles. After screening, 33 studies that met the entry and exit criteria were selected for the final review. We used Stata v. 14 and SPSS v. 22 applications to perform meta-analysis.

Results In the studies, $40.35 \%$ of the participants were older women and the rest were older men with a mean age of $67.80 \pm 6.44$ years. The mean score of death anxiety was higher in men than in women, and the elderly living in nursing homes had the highest score (11.8). Studies were categorized into three sections: comparison ( 3 studies), intervention (11 studies), and factors affecting the death anxiety (18 studies). The results of meta-analysis showed no significant relationship between spiritual therapy and reduction of death anxiety in the elderly $(P>0.05)$. In studies with spiritual and behavioral therapies, the heterogeneity was significant and, thus, a significant positive relationship was observed between the reduction of death anxiety and these treatments methods.

Conclusion The death anxiety level of the elderly in Iran is low. Religious beliefs, hopes for the intercession of imams and religious leaders, and hopes for freedom from the hardships of worldly life seem to have contributed to low death anxiety in Iran. Different death anxiety scores have been reported for older men and women in Iran may be due to the effect of culture, religion, and traditions, the difference in the roles of men and women, and even the expression of fear and anxiety. Most of men have less tendency to express their emotions, including fear, while women are more likely to express their feelings. Behavioral and spiritual interventions lead to a decrease in the elderly's death anxiety through affecting their finding meaning in life.

\section{* Corresponding Author:}

Masoudeh Babakhanian, PhD.

Address: Social Determinants of Health Research Center, Semnan University of Medical Sciences, Semnan, Iran.

Tel: +98 (912) 4312158

E-mail: babakhanian.m@gmail.com 


\section{Extended Abstract}

\section{Introduction}

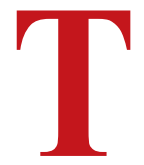

he issue of aging in recent years as has attracted the attention of experts, policymakers and even public. Reports show that, by the next 30 years, the percentage of aged population will reach approximately $26 \%$ of the total population [1]. One of the most common mental disorders in old age is death anxiety. Death anxiety refers to the thoughts, fears and feelings about the last days of life that a person experiences throughout life [2]. Death anxiety includes awareness of the importance of death and the types of beliefs, attitudes, images, and thoughts about death and what happens after death [4]. Given that during the past years, several studies have been conducted in the field of death anxiety of the elderly, this systematic review study aims to identify the death anxiety prevalence and effective interventions in reducing death anxiety in the Iranian elderly.

\section{Methods}

The present study is a systematic review of studies related to death anxiety of the elderly in Iran published in Persian from 2011-2019. In this regard, a search was conducted in national online databases including SID, Irandoc, Iranmedex, Magiran, IDML, ElmNet, and Civilica as well as Google Scholar using the keywords: "Death anxiety", "older adult", "fear of death" "elderly" and "Iran" in Persian. The target population included the entire aged population in Iran both men and women. The studies that had a standard structure covering type of service or intervention, aspect of service (living in nursing homes, community, daycare centers), client groups (older adults, retired adults), and professional role or task (death anxiety); and studies that were correlated, cross-sectional or had psychological interventions using behavioral therapy or spiritual therapy with and without a control group published from 2011 to 2019 were included in the study. Studies conducted in other fields (not on older people), theoretical studies, unpublished studies, and those published in other languages were excluded from the study.

Qualitative evaluation was used for interventional studies using the Cochrane modified checklist. For correlational and cross-sectional studies, the STROBE Checklist was used. First the data were saved in "dta" format. Then, the metan command of STATA software was used for metaanalysis. In order to check the publication bias, Begg's test was used. In correlational studies, the significance level was $\mathrm{P}>0.05$ and the confidence interval was -302.38 to -328.68 , indicating the absence of publication bias. In quasi-experimental studies, $\mathrm{P}$ value was also greater than 0.05 and the confidence interval was in range of -0.083 to -0.0089 . Due to the significance of Kendall's tau value, there was a significant relationship between the estimated value and the sample size. This indicates the existence of publication bias. In order to find the heterogeneity factor, meta-analysis of subgroups was performed. For this purpose, studies were divided into two groups; the first group measures the effect of behavioral therapies on reducing death anxiety and the second group measures the effect of spiritual therapy on reducing death anxiety. For data analysis, SPSS v. 22 software and STATA v. 14 software were used.

\section{Results}

Thirty three articles were included in the study and evaluated. Findings were divided into three sections: comparison (3 articles), intervention (11 articles) and factors affecting death anxiety in Iranian elderly (18 articles). In these articles, $40.35 \%$ of the participants were female and the rest were male with a Mean \pm SD age of $67.80 \pm 6.44$ years. The interquartile range of death anxiety was greater in men than in women, and the elderly living in nursing homes had the highest mean score of death anxiety (11.8). In correlational studies, death anxiety in the elderly had no statistically significant relationship with demographic variables, depression, social support, religious attitude and social intelligence. It had a significant positive relationship with external control, cognitive fusion, mental disorders and cognitive avoidance, while had a significant negative relationship with spiritual health, perception of aging, religious orientation, emotional intelligence, thanksgiving, external religious orientation, internal religious orientation, spiritual intelligence, spiritual well-being, social support, source of internal control, problem-focused coping, emotion-focused avoidance coping, quality of life, mental well-being, spiritual health, mindfulness, resilience, attachment to god, psychological well-being, religiosity, meaning of life, and aging. The results of comparative studies showed that death anxiety was significantly different between single and married older men, but there was no significant difference between single and married older women. Death anxiety scores of the elderly with pessimistic life orientation were significantly higher than that of those with optimistic life orientation. In 7 studies, the association between spiritual therapy and the reduction of death anxiety was reported. Although there was no heterogeneity between studies, but due to the lack of a significant level ( $\mathrm{P}=0.919)$, the results showed that there was no positive and significant relationship bet ween spiritual therapy and reduction of death anxiety in the elderly.

\section{Discussion and Conclusion}

Although different tools report different levels of death anxiety in the elderly, it seems that the death anxiety in the 
elderly in Iran is low. Older women are more likely to have death anxiety than older men, and the elderly living in nursing homes have more death anxiety than the elderly living at own home. Behavioral therapies have significant effect on reducing death anxiety in the elderly. Numerous variables are effective in reducing the anxiety of death in the elderly. By being aware of it, policymakers can reduce the phenomenon of death anxiety in the elderly in Iran. It is recommended that a review of studies published in other languages in the field of death anxiety in the elderly and its results be compared with the results of the present study.

\section{Ethical Considerations}

\section{Compliance with ethical guidelines}

This study was approved by the Research Committee of Mashhad University of Medical Sciences.

\section{Funding}

This research did not receive any grant from funding agencies in the public, commercial, or non-profit sectors.

Authors' contributions

All authors contributed equally in preparing this article.

\section{Conflicts of interest}

The authors declare no conflict of interest. 


\author{
اضطراب مرتى در سالمندان ايرانى: يك مرور نظاممند و مثاآناليز

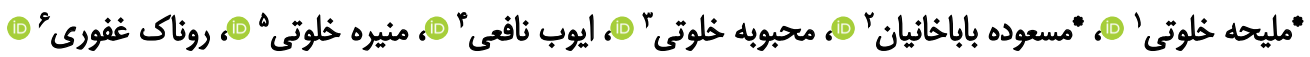 \\ 1. كروه مددكارى اجتماعى، دانشكده علوم بيرايزشكى، دانشكاه علوم يزشكى مشهيه، مشهله، ايران.

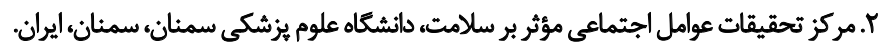

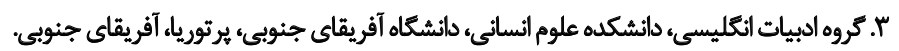

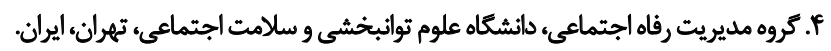

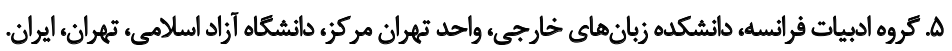

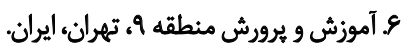

\begin{abstract}
حكين

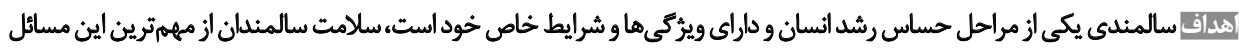

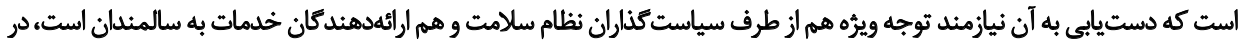

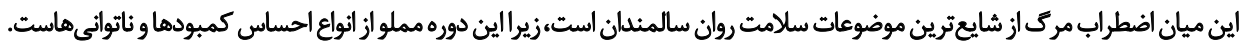

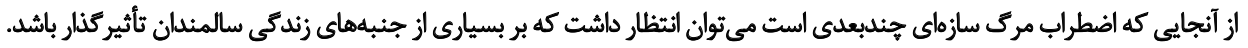

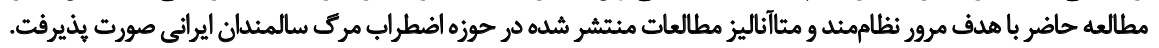

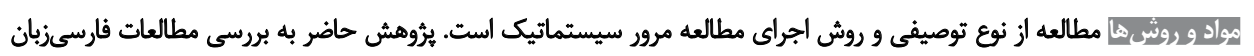

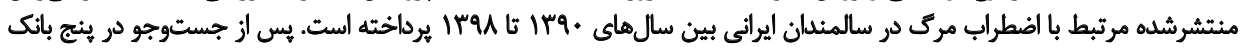

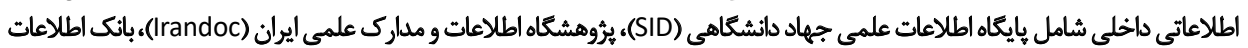

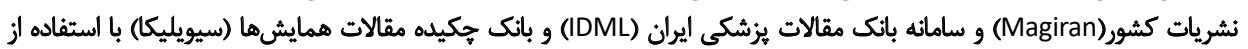

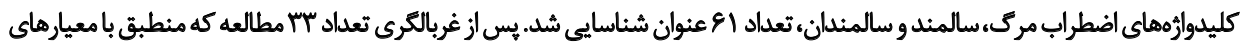

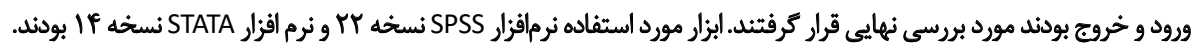

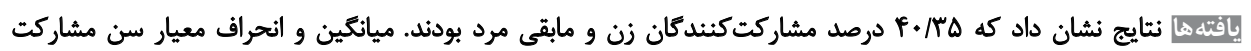

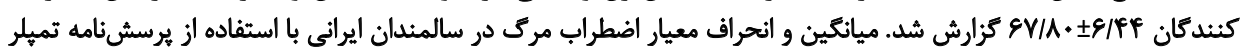

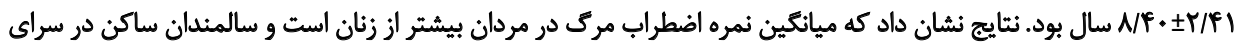

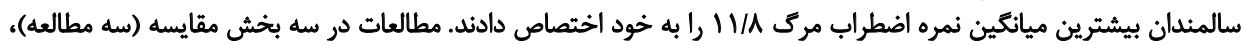

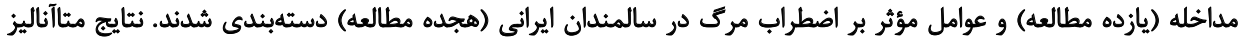

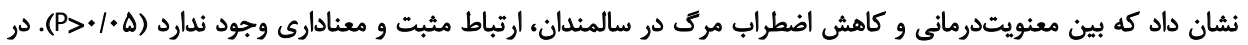

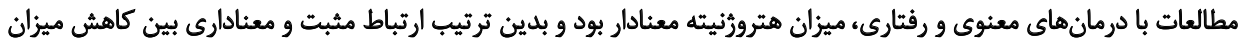

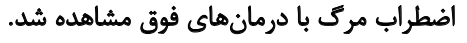

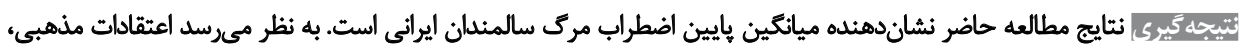

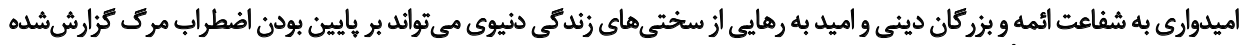

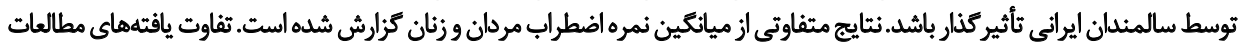

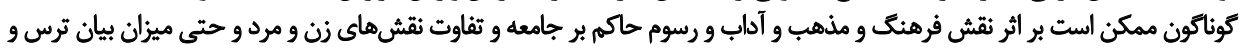

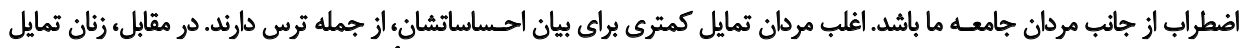

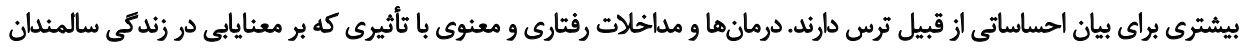

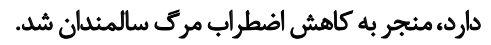

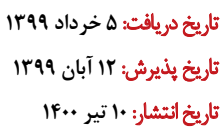

كليدواروها:

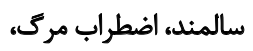
مرور نظاممند، متآنآليز 
سالمندان تأثيركذار باشد [11]]. اين ترس ممكن است از مقلمه

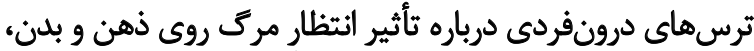

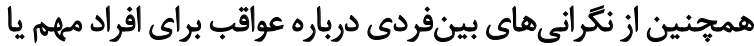

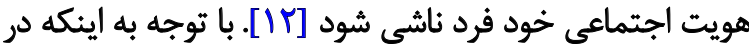

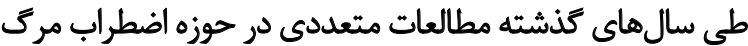

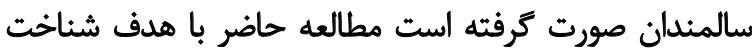

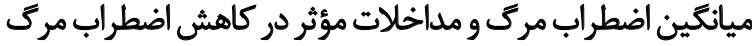
در سالمندان ايرانى به صورت يك مطالعه نظامدار انجام گرفت.

\section{ورش مطالعه}

مطالعه حاضر يك مطالعه توصيفى و به لحاظ روش اجرا مرور

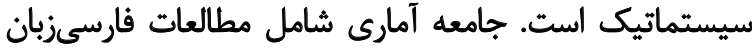

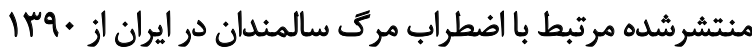
تا منتشرشده در حوزه اضطراب مرك سالمندان ايرانى انجام كرفت.

\section{استراتثى جستوجو}

براى دسترسى به مطالعات مربوط به اضطراب مركى سالمندان

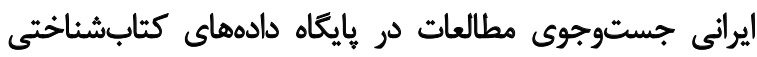

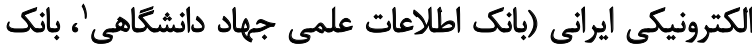

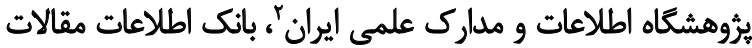

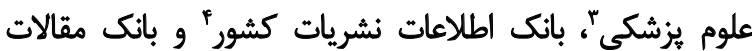

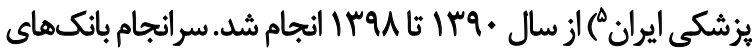

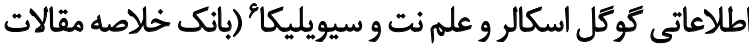

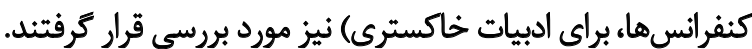

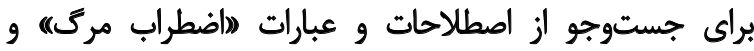
("سالمنده) يا (اترس از مرك)) و ("سالمندان) و ((ايران) استفاده شد.

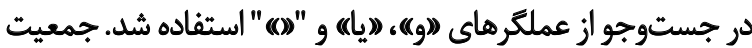

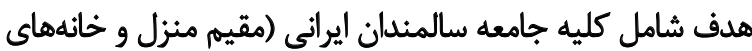
سالمندان) در هر دو جنس (زن و مرد) بود.

\section{معيار ورود وخروج و انتّناب مطالعات}

هدف يروهش مرور سيستماتيك اضطراب مرك در سالمندان ايرانى بود. بر اين اساس مطالعات فارسىزبانى كهد داراى ساختاري

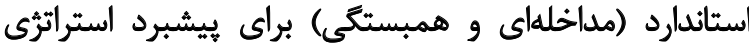
يُروهش بودند وارد مطالعه شدند. اين ساختار استاندارد شامل برد

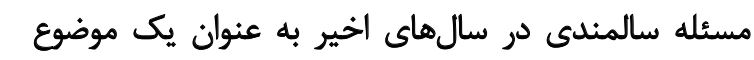

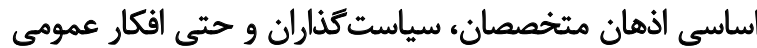

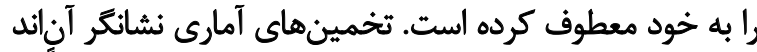

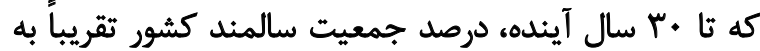

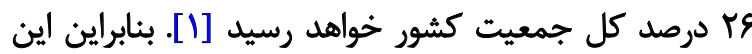

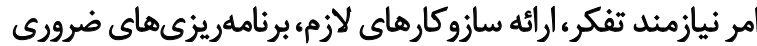

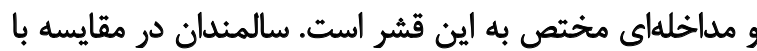

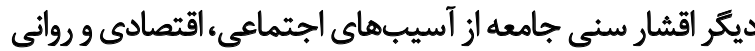

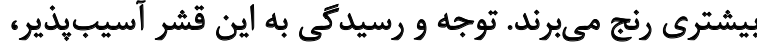

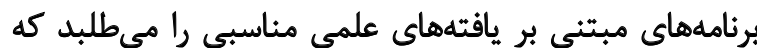

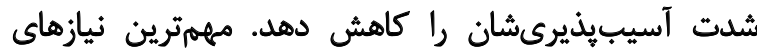

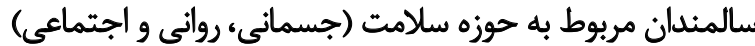

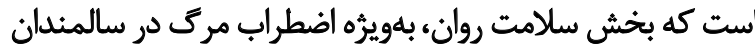
جزو موضوعاتى است كه كمتر به آن يرداخته شده است.

از شايعترين اختلالات روانى دوران سالمندى، اضطراب مرى

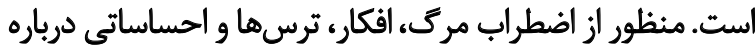

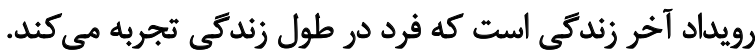

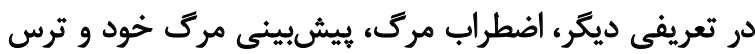

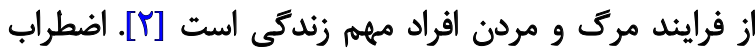

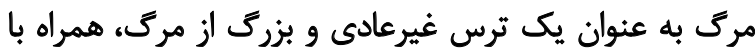

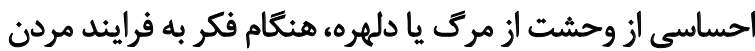

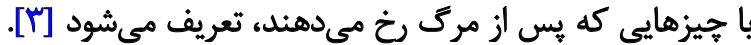

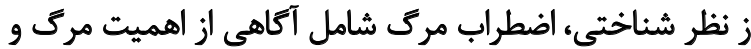

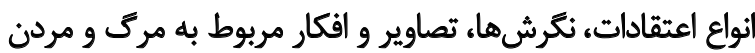

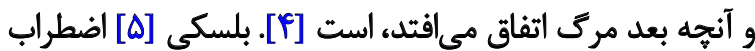

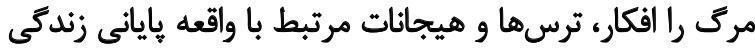

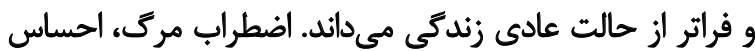

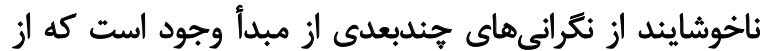

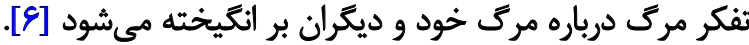

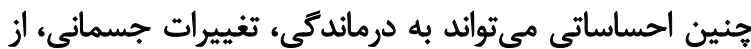

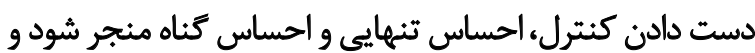

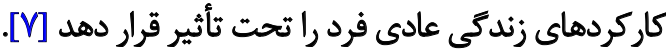
اضطراب يكى از مسائل شايع دوره سالمندى است؛ زيرا اين

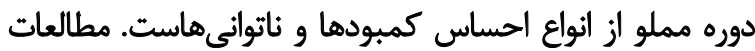

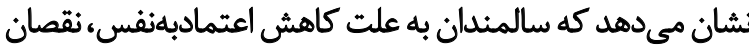

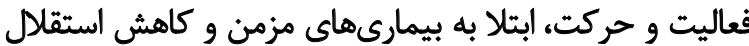

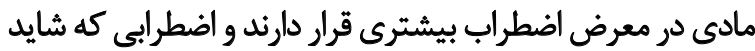

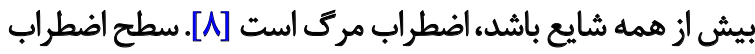

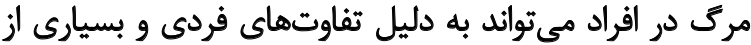

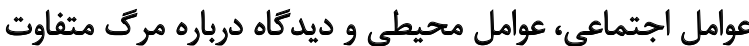

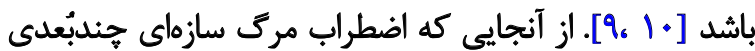
است مىتوان انتظار داشت كه بر بسيارى از جنبهائهاى زندكى إنى 


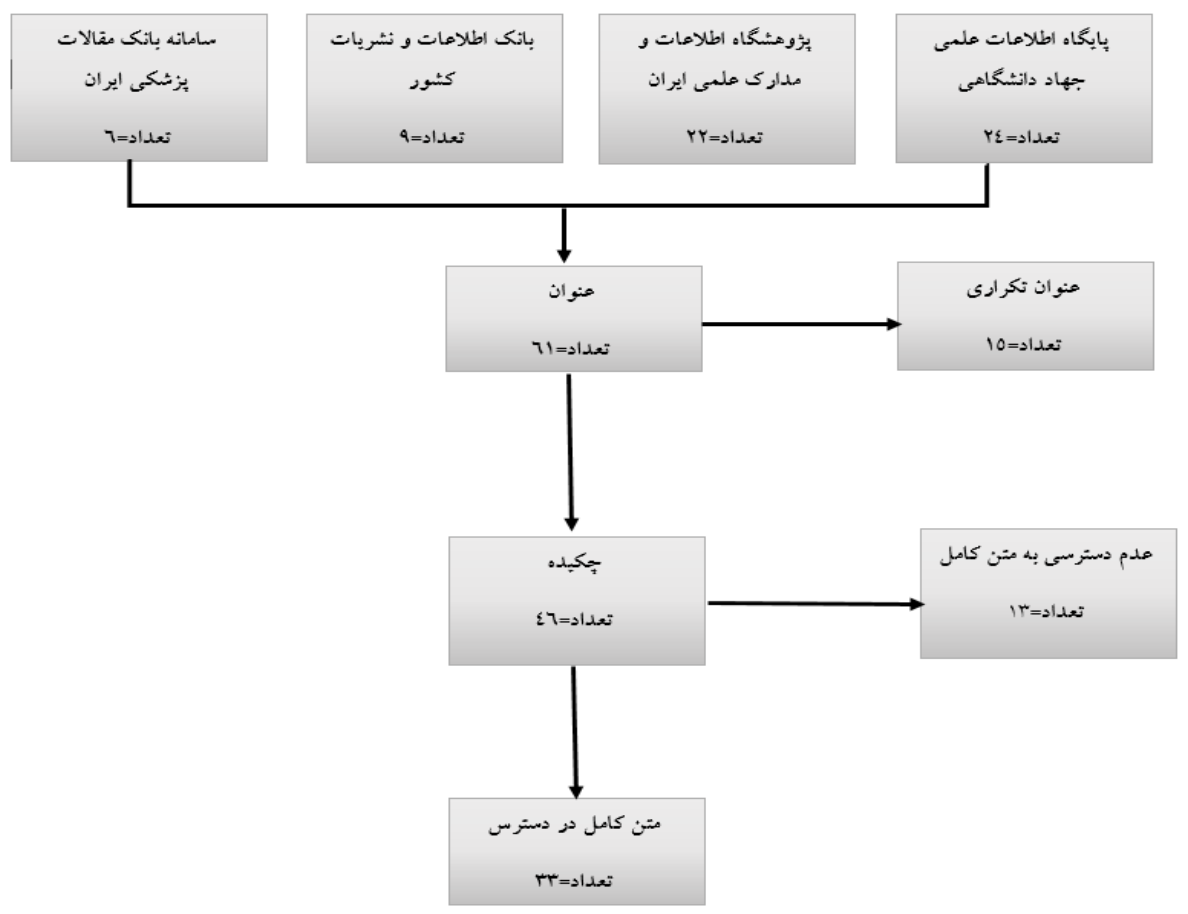

少

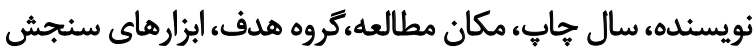

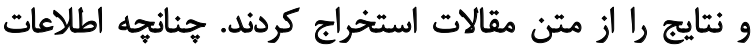

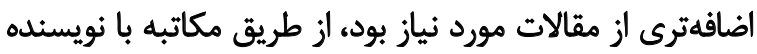

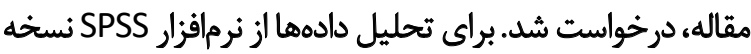

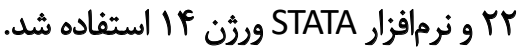

$$
\text { الوزيابى كيفى و سوكّرايیى انثشار }
$$

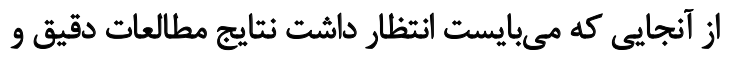

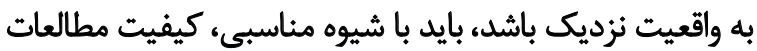

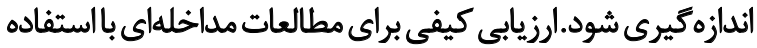

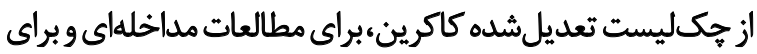

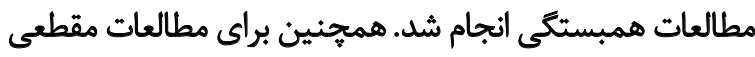

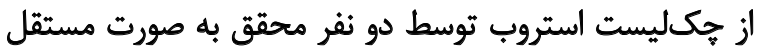

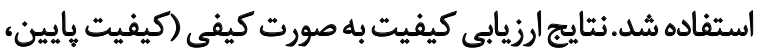

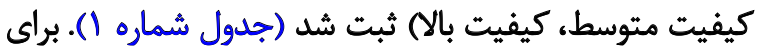

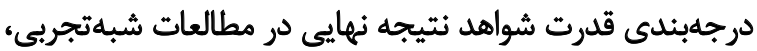

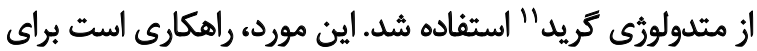

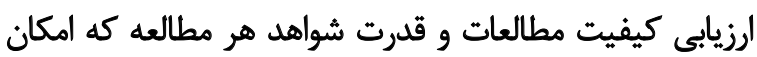

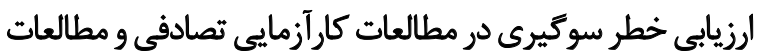

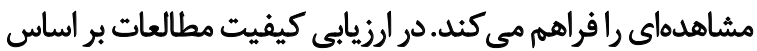

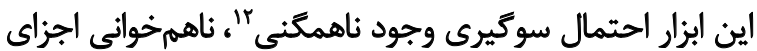

11. Grade

12. Inconsistency
تصوير ا. فلوجارت غربال مطالعات بر اساس استاندارد يريسما

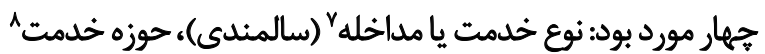

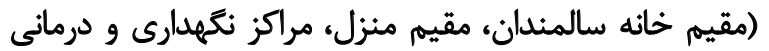

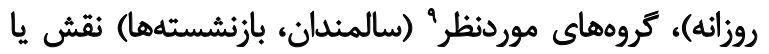

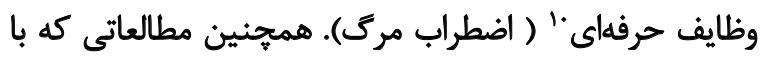

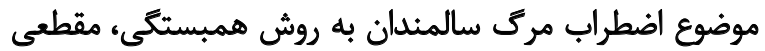

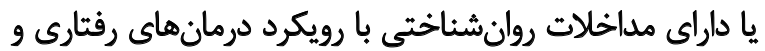

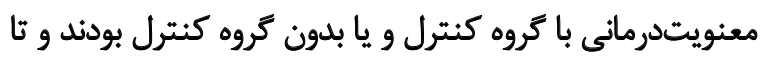

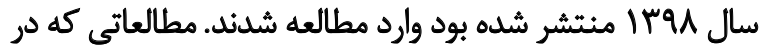

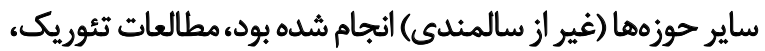

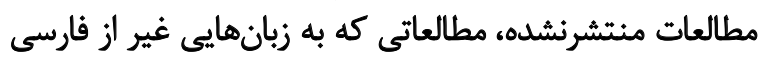
منتشر شده بودند به مطالعه راه نيافتند. در مرحله اول، جستوجو و بازيابى مقالات انجام كرفت. در

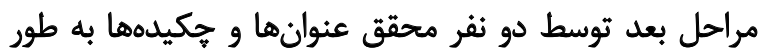

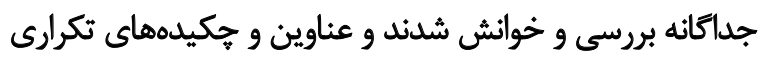

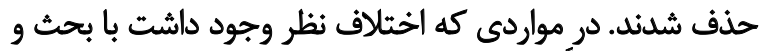

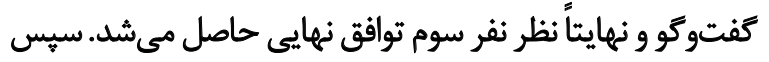

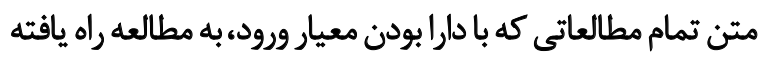

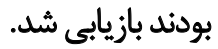
دو نفر محقق به صورت مستقل اطلاعات موردنياز، شامل نام

\section{Type of service or intervention}

8. Aspect of service

9. Client groups

10. Professional role or task 
مثآناليز

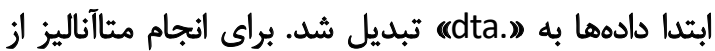

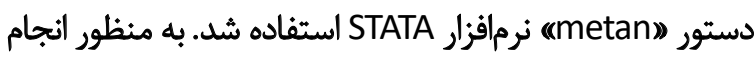

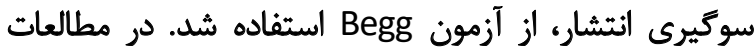

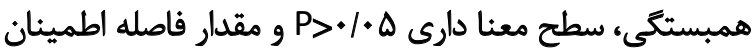
سوكرايى انتشارد/, سو

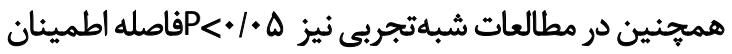

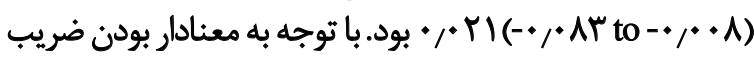

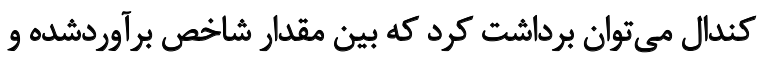

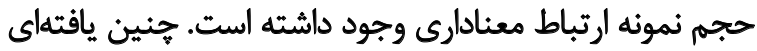
وجود سوكرايى انتشار را نشان مى دهد.

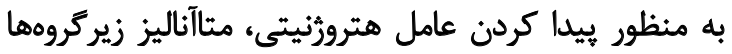

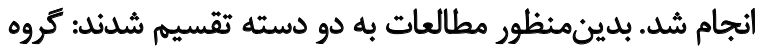

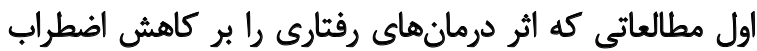

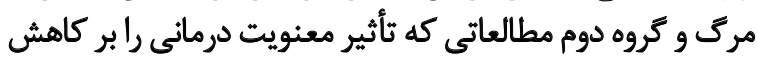
اضطراب مرك در سالمندان سنجيده بودند وائير معنويت درماني تحليل شراند
مطالعه" و عدم دقت "و و احتمال سوكيرى تعيين مىشود. به

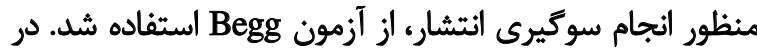

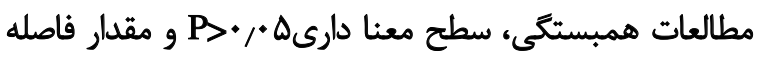

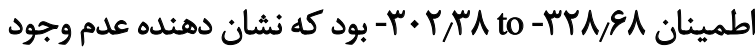

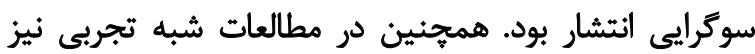

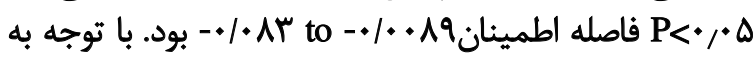

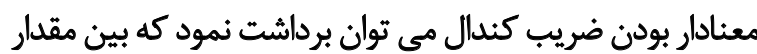

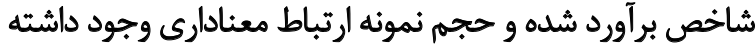

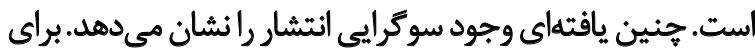

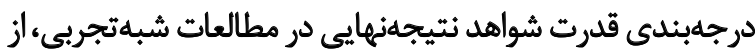

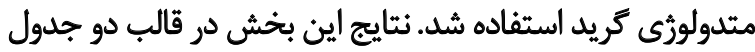

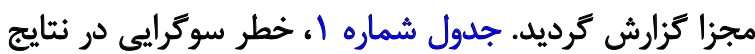

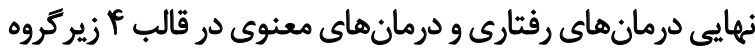

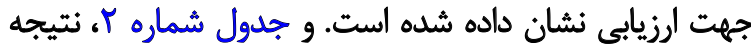

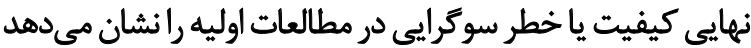

13. Indirectness

14. Imprecision

جدول شماره ا. كيفيت (خطر سوكرايي) در مطالعات نيمه تجربي بر اساس جك ليست كاكرين

\begin{tabular}{|c|c|c|c|c|c|}
\hline 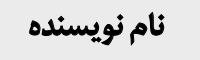 & كورسازى & كثترل مخدوقُّكُرها & ييكيرى حضور ييماران تا بايان درمان & وجود يايامد ثائويه & كيفيت \\
\hline حاجى عزيزى & خطر سوكرايى هايين & خطر سوكرايي بايين & خطر سوكرايى بالا & خطر سوكرايع يايين & بالا \\
\hline مجيدى & خطر سوكرايق هايين & حُطر سوكرايع مثونسط & خطر سوكرايي بالالا & حُطر سوكرايي بالا & 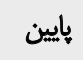 \\
\hline خدابخشيى & خطر سوكرايى بايين & خطر سوكرايي متوسط & خطر سوكرايى بالا & خطر سوكرايى بايين & متوسط \\
\hline كل محمديان & خطر سوكرايى بايين & خطر سوكرايى متوسط & خطر سوكرايى بالا & خطر سوكرايى بالا & 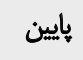 \\
\hline دشت بزركى & خطر سوكرائي هايين & خُطر سوكرايع مثوسط & مُطر سوكرايي بالا & خطر سوكرايع هايين & متوسط \\
\hline صلرى دميرجى & خطر سوكرايع جايين & خطر سوكرايي متوسط & خطر سوكرايى بالا & خطر سوكرايع بايين & 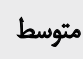 \\
\hline دهنان & خطر سوكرايع هايين & خطر سوكرايع متوسط & خطر سوكرايى بالال & خطر سوكرايي هايين & متوسط - مثط \\
\hline ولايى & خطر سوكرايى بايين & خطر سوكرايي متوسط & خطر سوكرايى بالا & خطر سوكرايي بالا & بإيين \\
\hline قربائعلى يور & خطر سوكرايى هايين & خطر سوكرايع متوسط & خطر سوكرايي بالا & خطر سوكرايي بالا & بايين \\
\hline غلاهى & خطر سوكرايى ثايين & خطر سوكرايى متوسط & خطر سوكرايى بالا & خطر سوكرايى بايين & متوسط \\
\hline خدابخش يير كلانى & خطر سوكرايي إيايين & خطر سوكرايى متوسط & خطر سوكرايع بالا & خطر سوكرايي يايين & متوسط \\
\hline
\end{tabular}


جدول r. ارزيابي و سنجش(GRADE) كيفيت شواهد نتايج ييامدهاي مداخلات در مطالعات

\begin{tabular}{|c|c|c|c|c|c|c|c|c|c|}
\hline \multirow[b]{2}{*}{ كيفيت } & \multirow{2}{*}{ أندازه اثر } & \multicolumn{2}{|c|}{ تعداد بيماران } & \multicolumn{4}{|c|}{ نمره ارزيابى } & \multirow[b]{2}{*}{ مطالعات } & \multirow[b]{2}{*}{ ييامد } \\
\hline & & $\begin{array}{l}\frac{k}{k} \\
\frac{k}{\xi}\end{array}$ & $\frac{\varepsilon}{\frac{\varepsilon}{\xi}}$ & $\begin{array}{l}6: \\
6 \\
b\end{array}$ & C. & $\frac{5}{6}$ & $\begin{array}{l}G \\
\text { है } \\
\text { है } \\
\text { है }\end{array}$ & & \\
\hline هتوسط & $\begin{array}{c}r / \cdot W \\
(95 \% \mathrm{Cl}: 1.380-2.775)\end{array}$ & q. & 9. & بالا & بايين & بالا & هتوسط & $r$ & اثر ماهش اضطراي رفتارى براب \\
\hline بايين & $\begin{array}{c}\text { Y/MY } \\
(95 \% \mathrm{Cl}: 0.73-3.70)\end{array}$ & M. & ri. & بايين & بايين & بالا & متوسط & $\checkmark$ & أثر درمانهاي معنوى براب \\
\hline
\end{tabular}

䂪

(هجده مطالعه) تنظيم شد. مطالعات موردارزيابي بين سال هاي

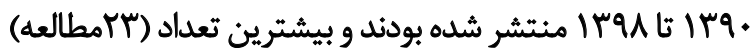

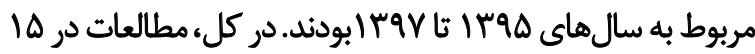
شهر انجام شده است (جلدول شماره مأه).

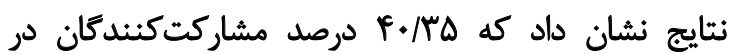

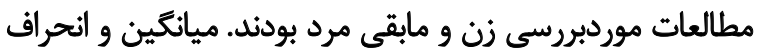

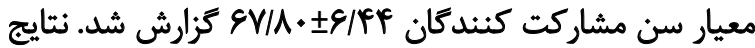

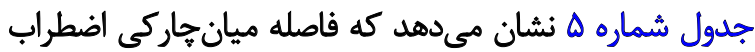

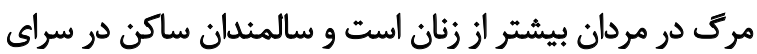

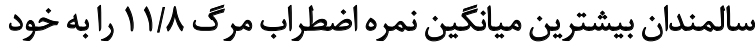

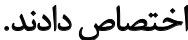

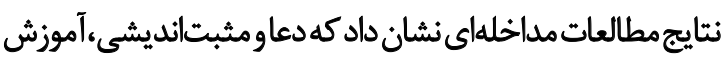

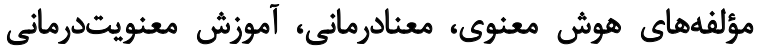

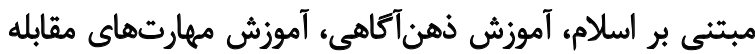

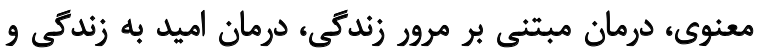

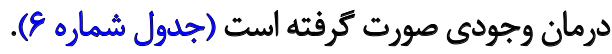

نتايج نشان مي دهد كه در مطالعات مريوط به بررسي همبستيكى،

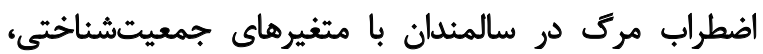

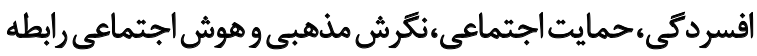
معنى دار آمارى نداشت. اضطراب مرى سالمندان با منبع كنترل

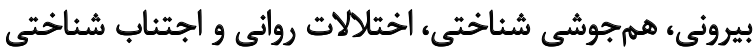

(جدول شماره |). نتايج در جدول شماره | |و ب نشان داده شده

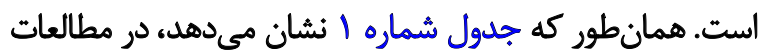

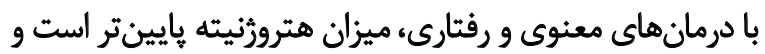

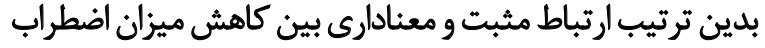

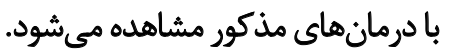

براى درجهبندى قدرت شواهد نتيجه نهايى در مطالعات

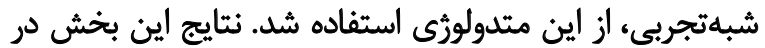

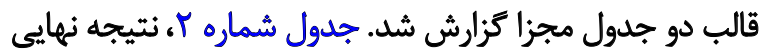

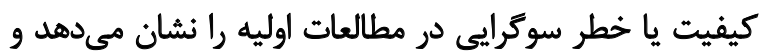

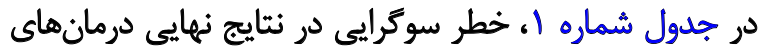

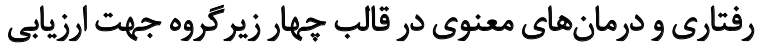
نشان داده شده است.

Laت̣̂ㄴ

با جستوجوى كليد وارٔهاى موردنظر در بانكهاى اطلاعاتى

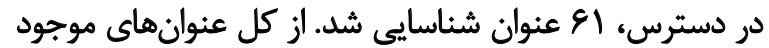

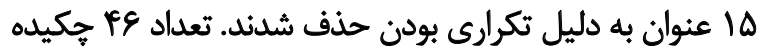

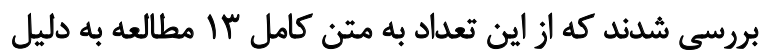

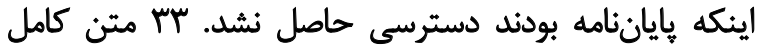

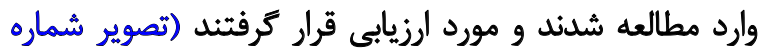
(). يافتهها در سه بخش مقائه مقايسه (سه مطالعه)، مداخله (يازده

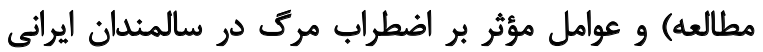

جدول ب. آناليز زير كروه داده هاى مطالعات شبه تجربى بر كاهش اضطراب مرك در سالمثدان

\begin{tabular}{|c|c|c|c|c|c|}
\hline سطح معنادارى & 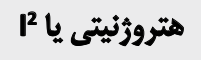 & ه 9 دروفاصله اطمينان & اندازه اثر (SMD) & تعد|د مطالعه & نوع مطالعه \\
\hline$<\cdot 1 . .1$ & $A V / T$ & TMQ -I/TA. & $r / W$ & $\checkmark$ & هطالعات با درمانهاي معنوى \\
\hline$<\cdot / . .1$ & $9 \% / 6$ & $r / N \cdot-\cdot M^{m}$ & $T / M$ & $r$ & مطالعات با درمانهاي رفتارى \\
\hline
\end{tabular}

证 


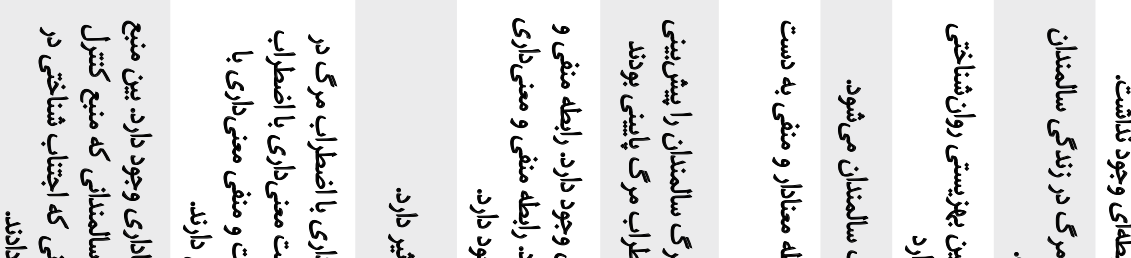

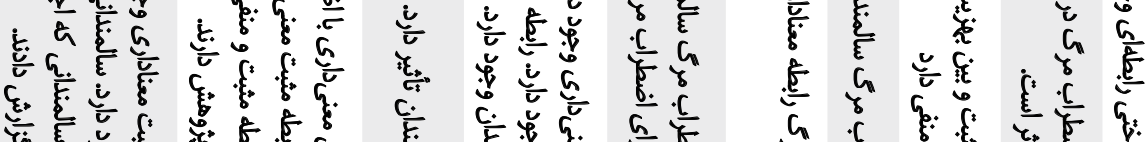

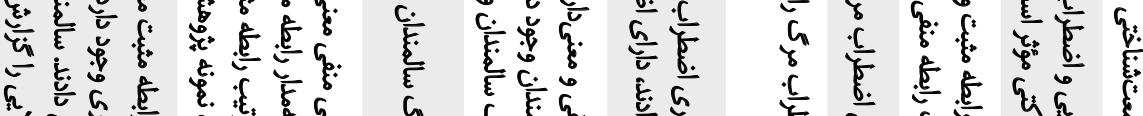

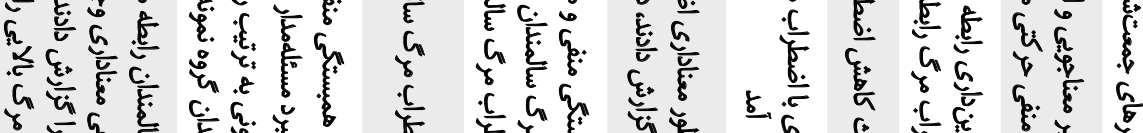

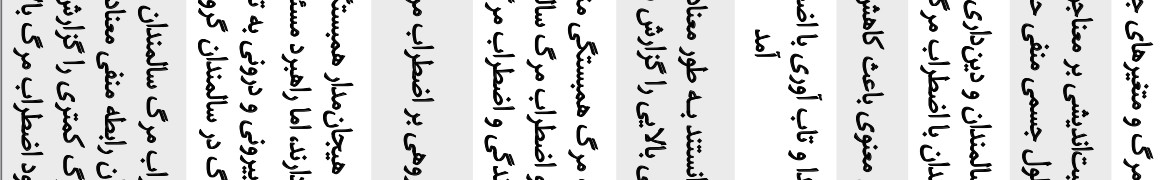

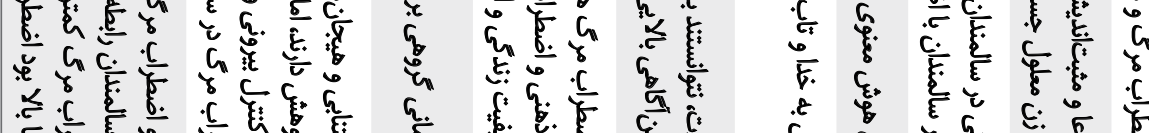

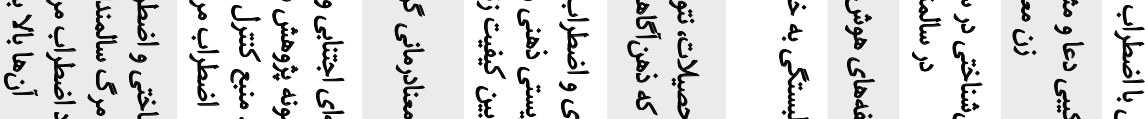

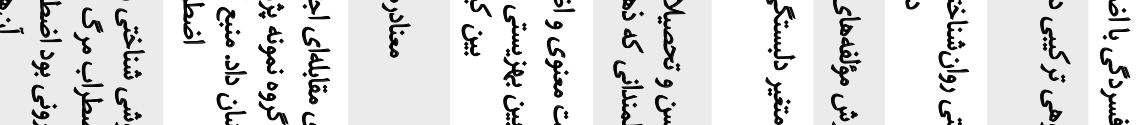

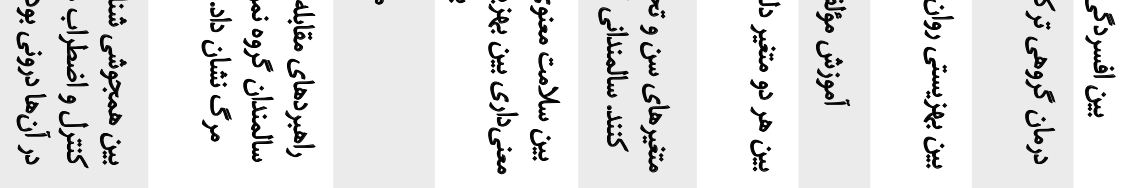

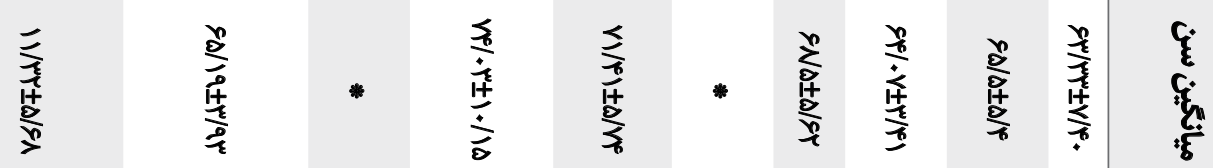

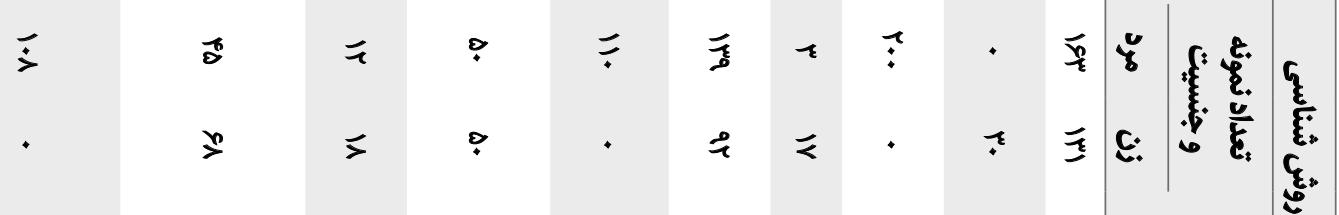

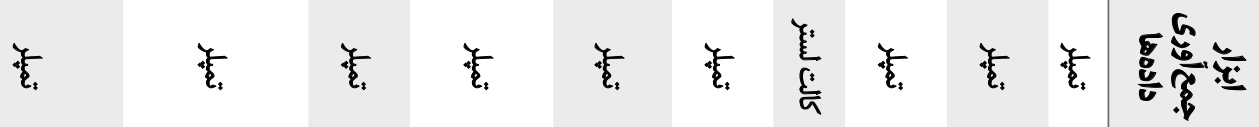

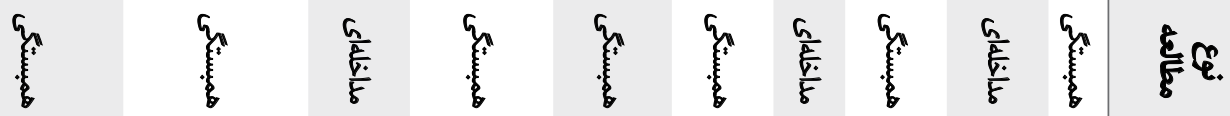

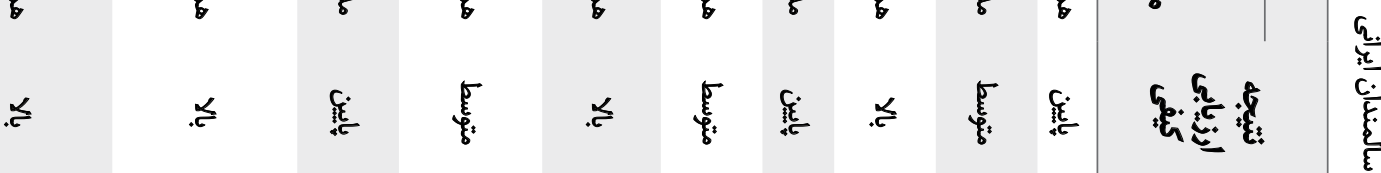

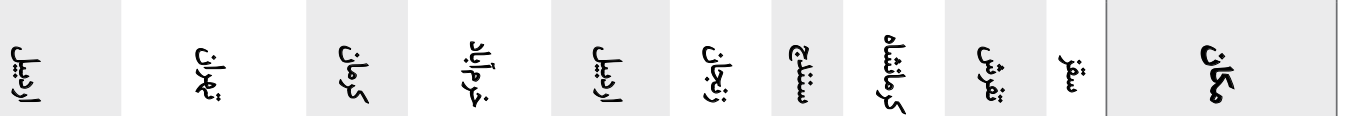

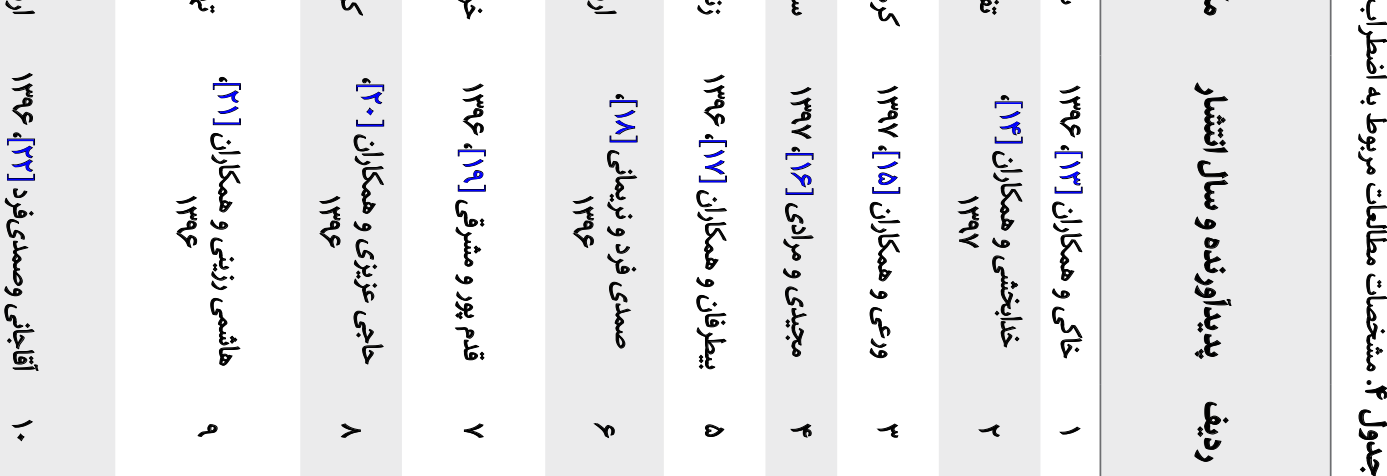



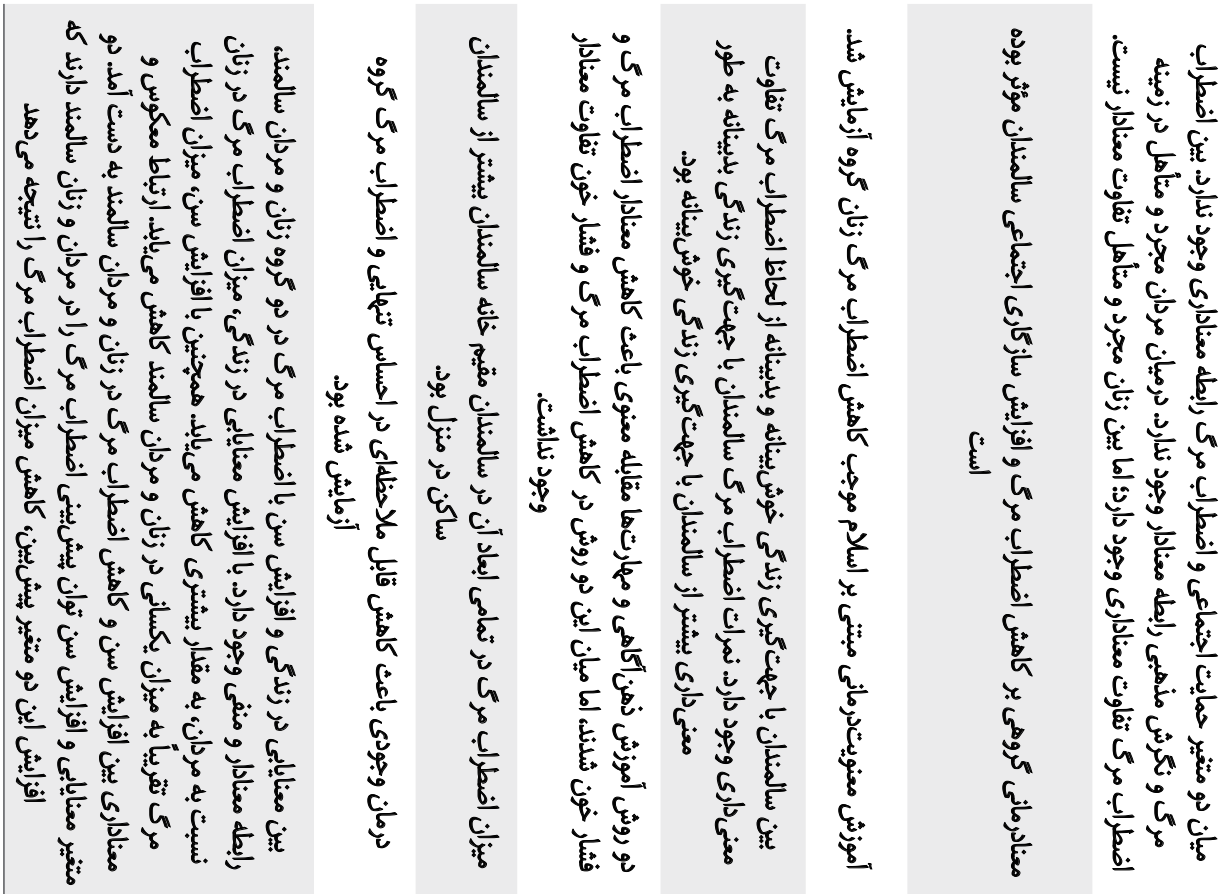

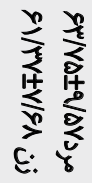

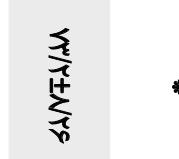

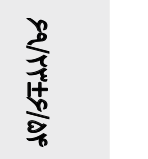

$\frac{5}{4}$

$\frac{\xi}{\xi}$

$\div$

$\div$

$\pm \quad \frac{2}{3}$

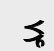

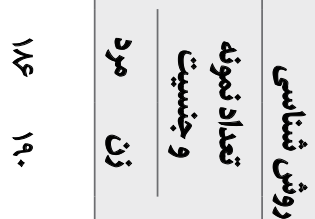

है

$\frac{\xi^{*}}{\frac{5}{a}}$

$\frac{5}{4}$

告

है

?

$\frac{a}{b}$

ह $\frac{a}{b}$

$\frac{a}{b}$

ह.

है?

s.

$\approx \quad \xi$

\&.

$\approx$

$\approx$

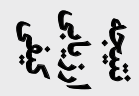

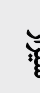

管案

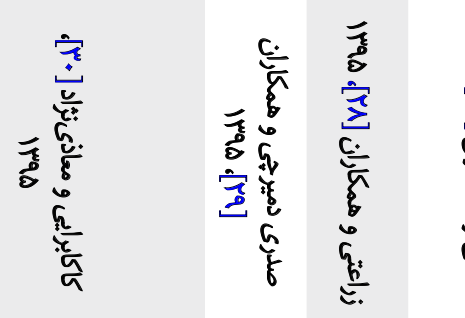

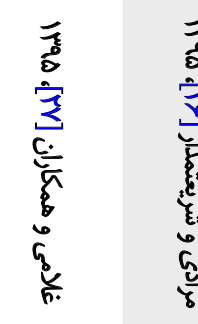

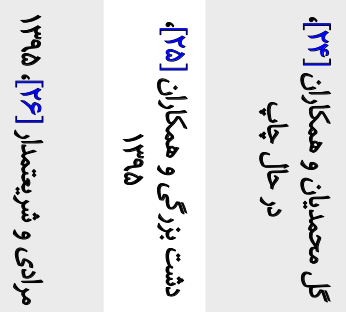

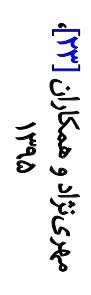

ç.

5

$z$

$\bar{\sigma}$

千

$=$

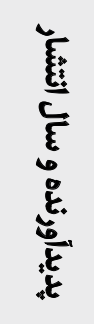

E. 


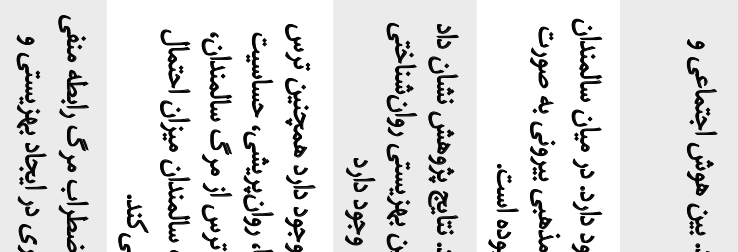

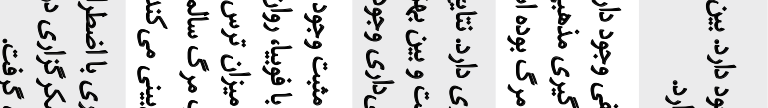

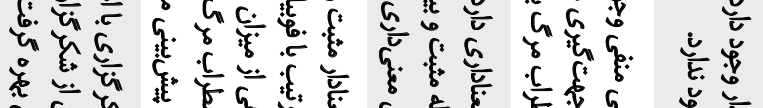
等

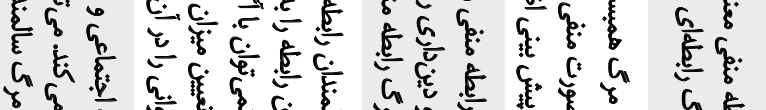

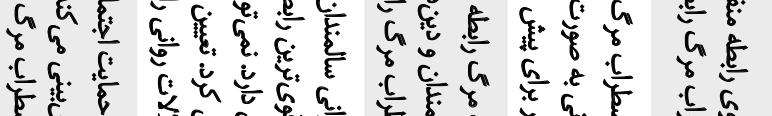
整

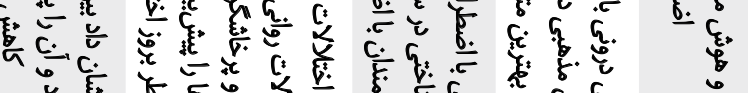

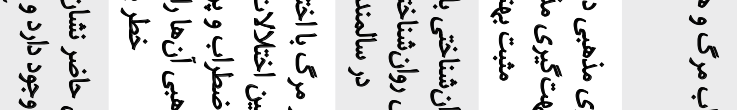

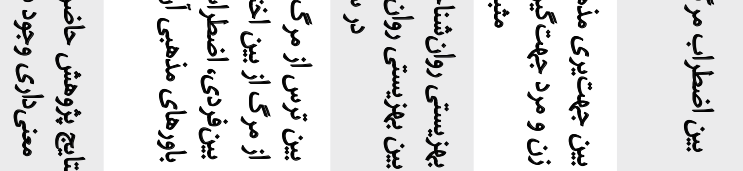
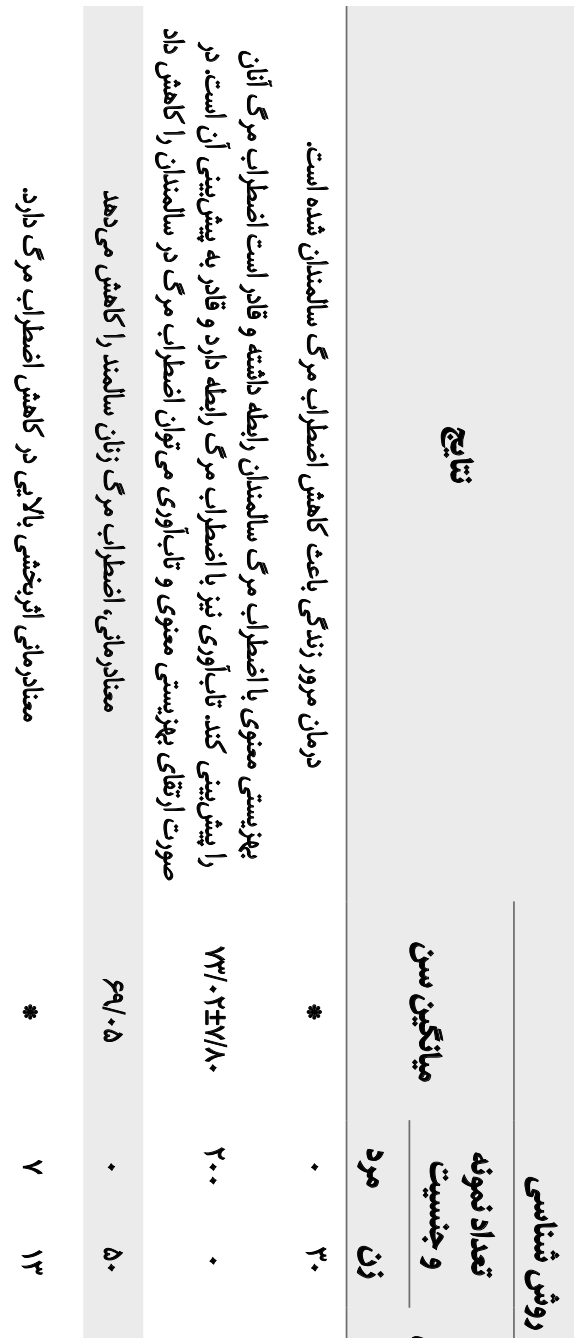

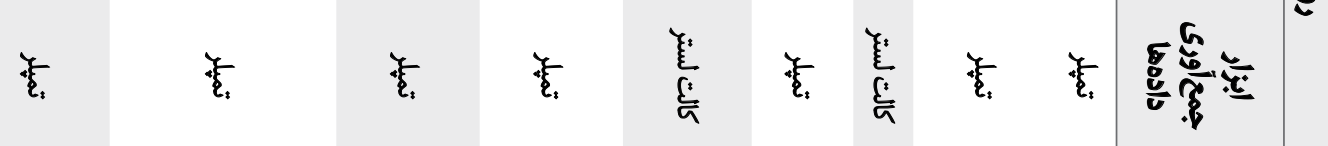

?

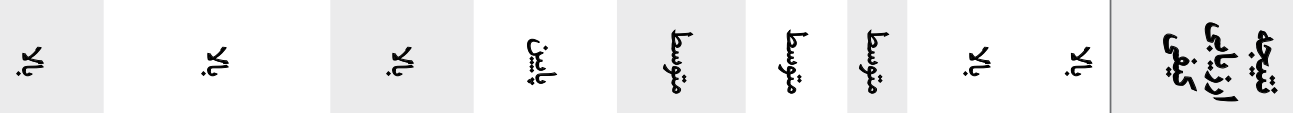

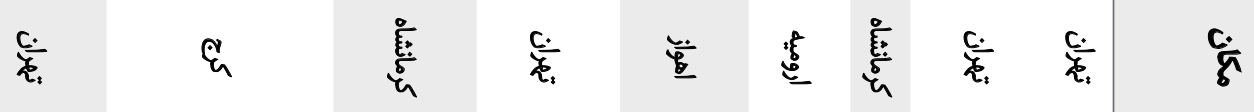

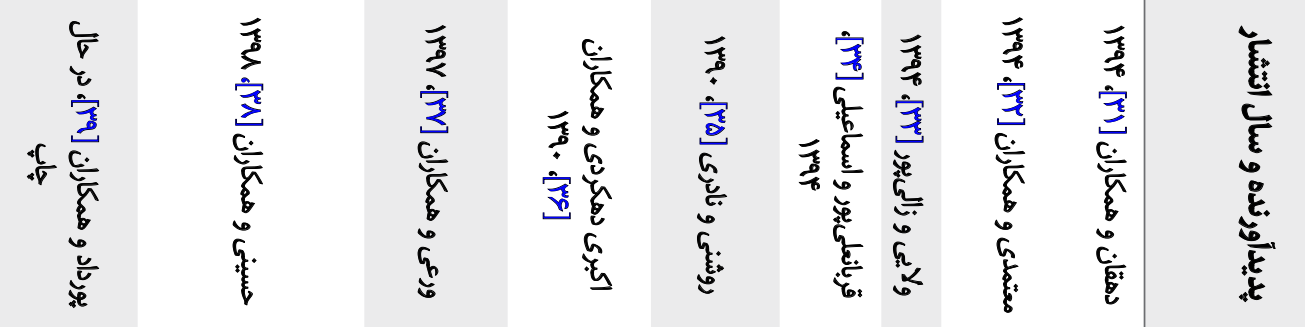
$z$
$\vec{b}$
$\bar{s}$
告
z
$z=?$
8 


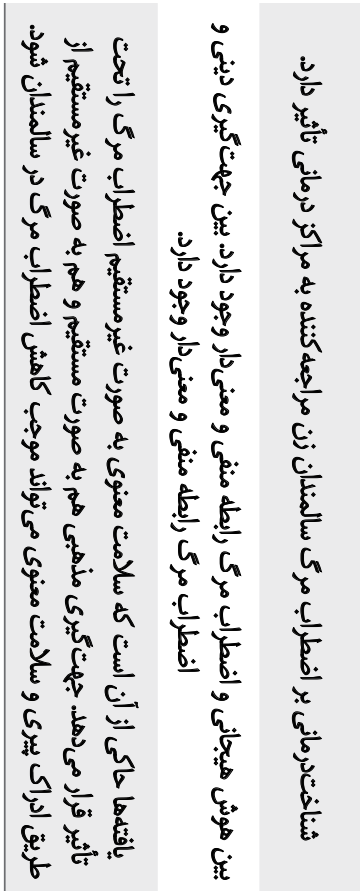

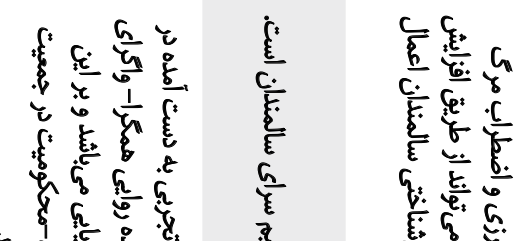

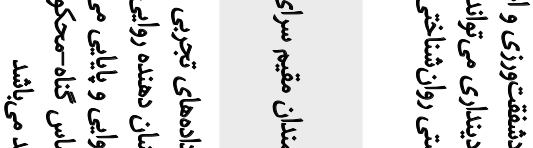

दह ह

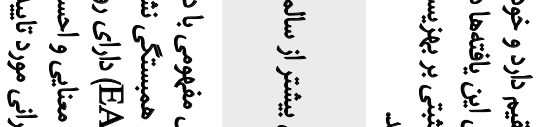
द.

है

$\stackrel{?}{\varepsilon}$

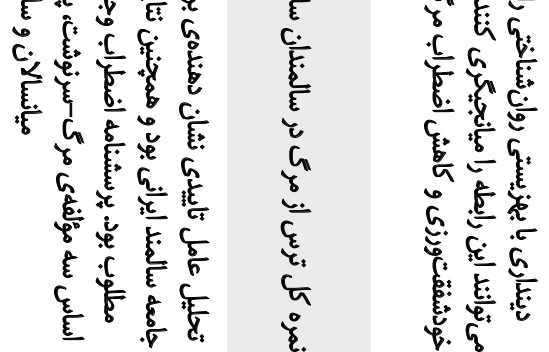

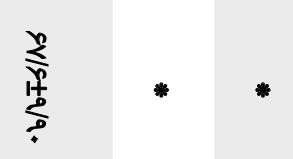

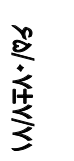

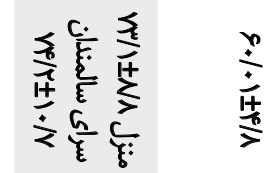

$\equiv \quad \xi \quad$ z

s

$\stackrel{+}{*}$

$\sqrt{5}$

$\bar{z}$

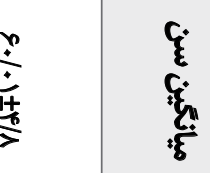

है है है

s.

$\frac{y}{\frac{6}{a}}$

c.

i 1

客善

$\xi$

$\frac{E}{E}$

E है

$\xi^{5}$

$\approx \quad .5$

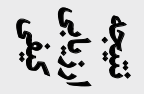

旁

.

c.

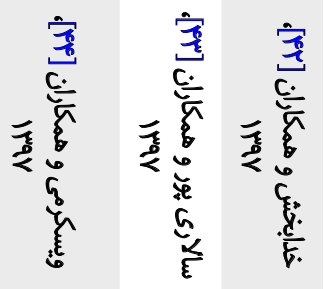

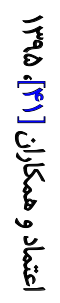

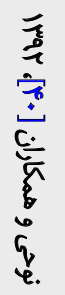

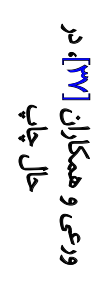

1

$?$

군

है


جدول ه. مشخصات جمعيتشناختى سالمثدان در مطالعات اوليه

\begin{tabular}{|c|c|c|}
\hline درصد(فراوانى)/ميانكين +انحراف معيار & \multicolumn{2}{|c|}{ مثغير } \\
\hline 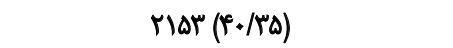 & نj & \multirow{2}{*}{ جنسيت فراوانى } \\
\hline $\operatorname{MIAY}\left(\Delta Q / P^{\circ}\right)$ & مرد & \\
\hline eV/A. $\pm R / P P$ & \multicolumn{2}{|c|}{ 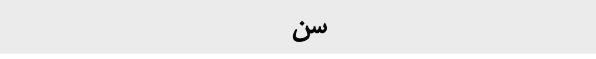 } \\
\hline NEV $(1 / 1 F)$ & 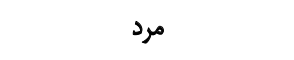 & \multirow{2}{*}{$\begin{array}{c}\text { اضطراب مرك ميانه (فاصله } \\
\text { (فياركى) }\end{array}$} \\
\hline$V / M I(M / M T)$ & نj & \\
\hline$q / 4 \cdot(r / e V)$ & ساكن منزل & \multirow{3}{*}{ محل نكهيدارى سالمند } \\
\hline$W / A\left(r / \varphi_{*}\right)$ & ساكن سراى سالمندان & \\
\hline$N Q I(Y / I T)$ & مراكز نكتهدارى و درمان روزانه & \\
\hline
\end{tabular}

\section{L}

بود.نتايج مطالعات مقايسهاى نشان داد كه اضطراب مرك در ميان

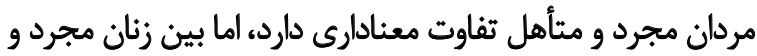

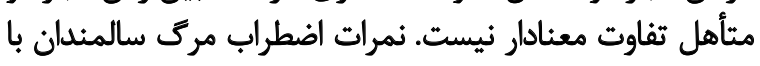

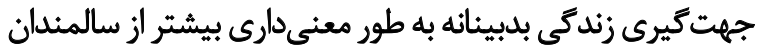

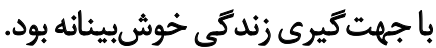

$$
\text { ارثباط معنويثدرماني با كاهش اضطراب مركى }
$$

در هفت مطالعه، ارتباط بين معنويتدرمانى و كاهش اضطراب

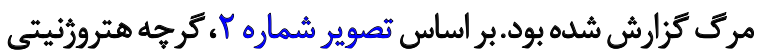

رابطه مثبت و معنى دار آمارى داشت. نتايج همجنين نشاندهنده

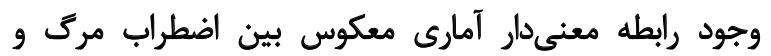

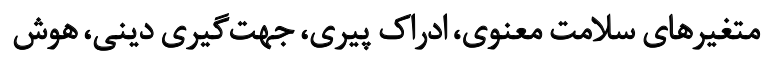

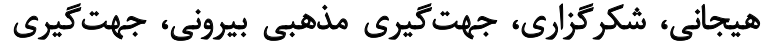

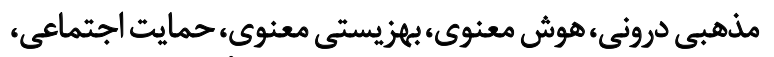

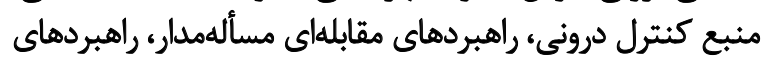

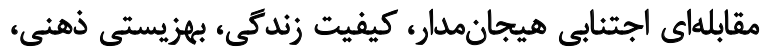

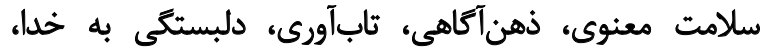

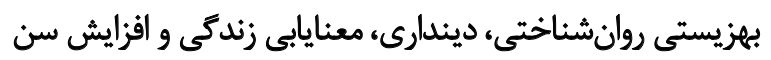

جدول \&. مداخلات انجام شده براى كاهش اضطراب مرك سالمندان

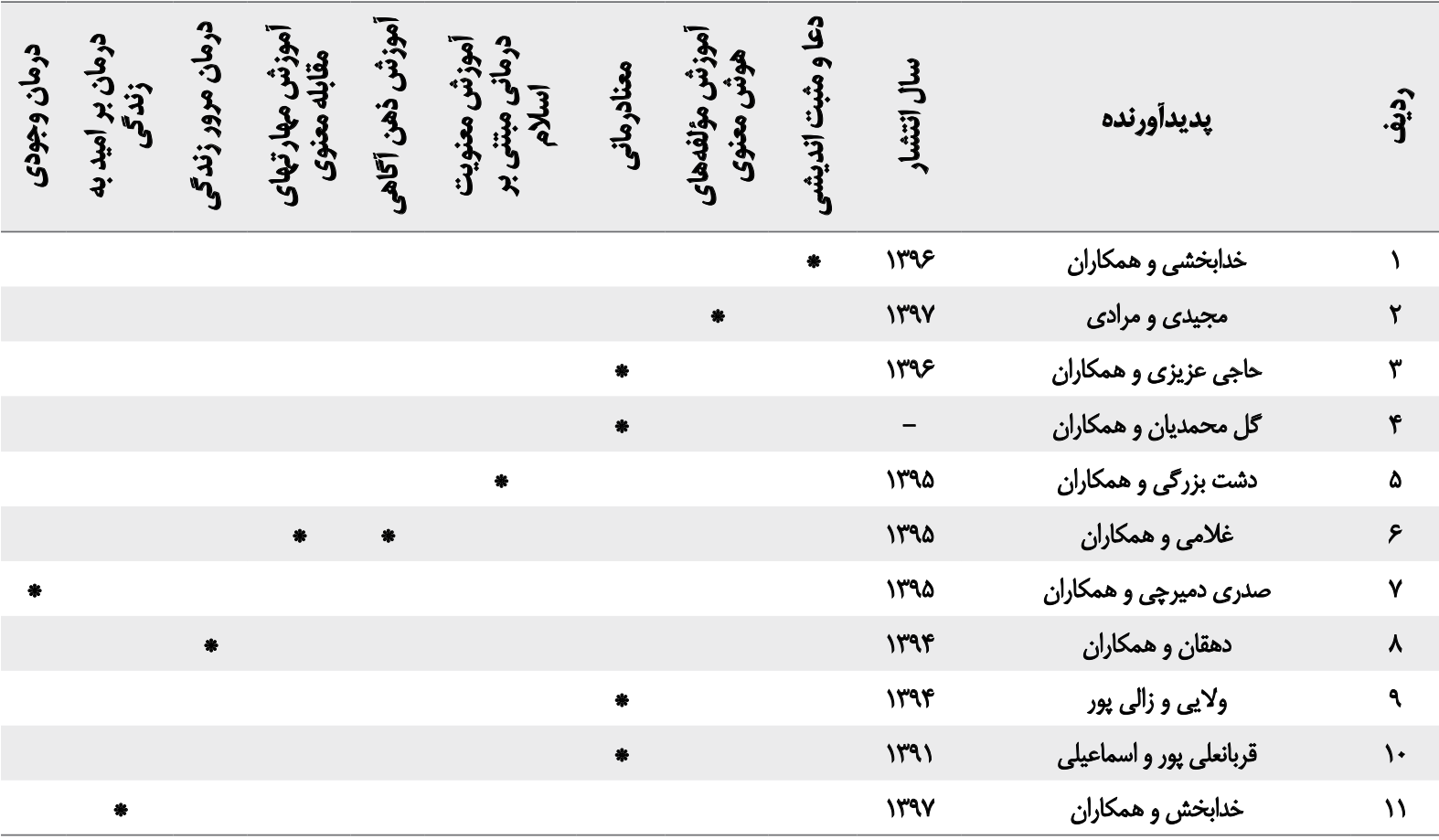

L 
Study

bitarafan (1396)

ghadampour (1396)

motamedi (1394)

payamvaredi (1397)

payamvarei (1398)

salaripour (1397)

viskarami (1397)

Overall (l-squared $=0.0 \%, p=0.914$ )

(n)
$\%$

ES $(95 \% \mathrm{Cl})$

Weight

7.70

$9.78(0.65,18.91) \quad 3.79$

$7.21(4.74,9.68)$

$8.57(3.42,13.72) \quad 11.90$

$8.46(3.19,13.73) \quad 11.38$

$8.10(1.75,14.45) \quad 7.84$

$8.34(0.77,15.91)$

\begin{tabular}{l}
$7.47(5.69,9.25) \quad 100.00$ \\
\hline
\end{tabular}

닌

تصوير r. نمودار همبستكى با فاصله اطمينان 9 هدرصد براى ارتباط معنويتدرمانى بر كاهش اضطراب مرى
Study

ID

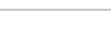

majidi (1397)

khodabakhshi (1395)

golmahammadian (1395)

dasht bozorgi (1395)

gholami (1395)

dehghan (1394)

valaei (1394)

ghorbanalipor (1391)

khodabakhsh pir (1397)

Overall (I-squared $=88.4 \%, \mathrm{p}=0.000$ )

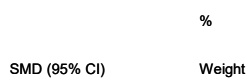

$1.50(0.93,2.08)$

$2.43(1.60,3.25)$

11.45

5.56

$3.18(2.41,3.95)$

6.41

$1.24(0.68,1.79) \quad 12.36$

$1.66(1.07,2.25) \quad 10.91$

$3.75(2.90,4.60) \quad 5.24$

$2.05(1.42,2.68) \quad 9.59$

$1.00(0.58,1.41) \quad 21.88$

$3.84(2.78,4.90) \quad 3.35$

$0.96(0.42,1.49) \quad 13.24$

$1.71(1.52,1.91) \quad 100.00$ 
اسجاماكر و همكاران نشاندهنده نبود وجود اختلاف معنادار

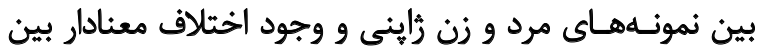

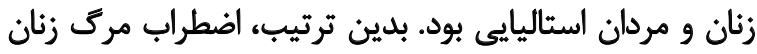

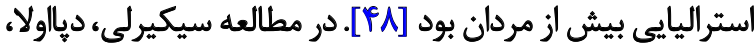

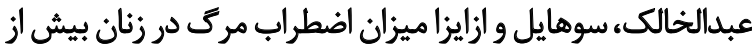

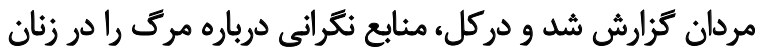

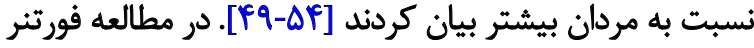

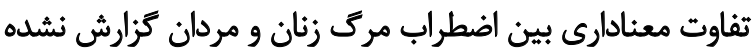

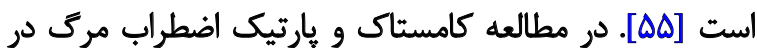

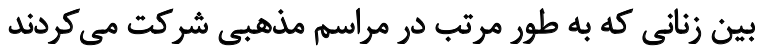

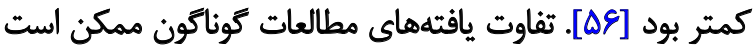

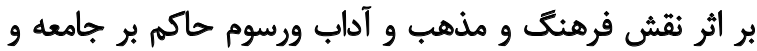

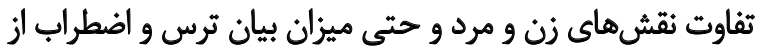

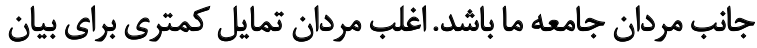

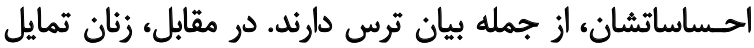

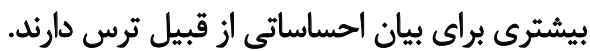
در مطالعه حاضر نتايج نشان داد كه سالمندان ساكن سراى

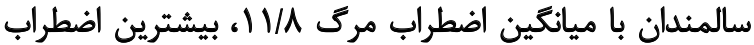

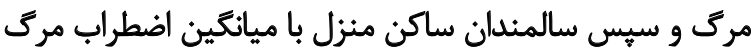

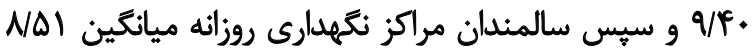

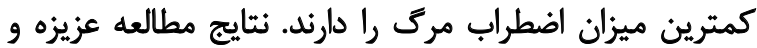

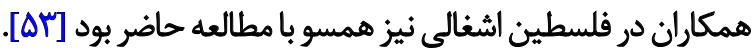

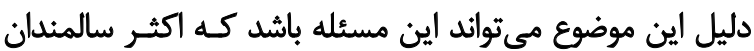

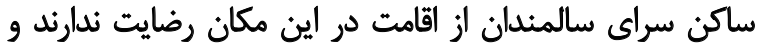

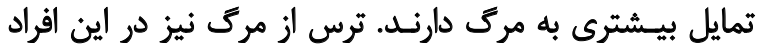

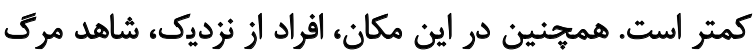

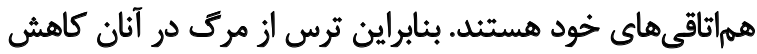

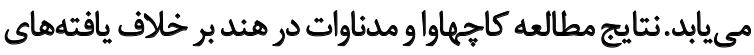

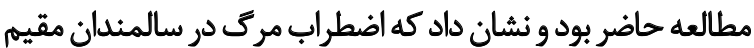

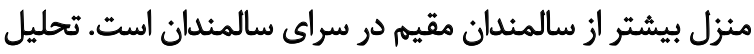

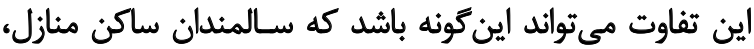

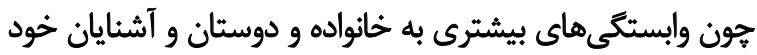
دارند، ترس از مرك نيز در اين افراد بيشتر است.

نتايج مطالعه حاضر نشان داد كه با افزايش سلامت معنوى،

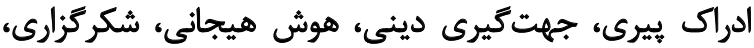

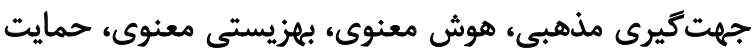

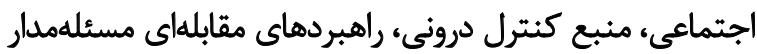

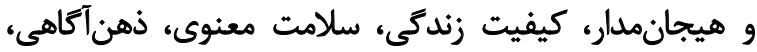

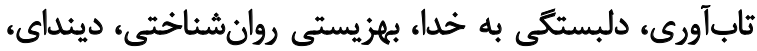

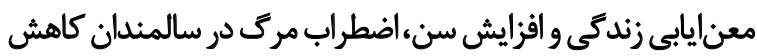

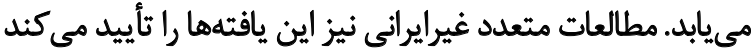

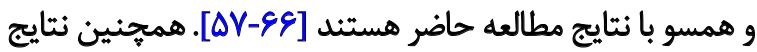

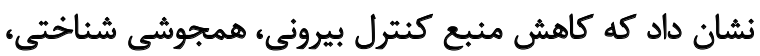
اختلالات روانى و اجتناب شناختى منجر به كادئ كاهش اضطراب

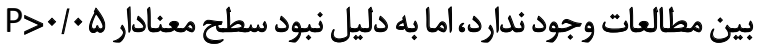

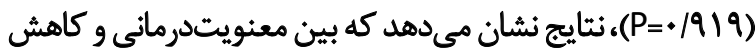

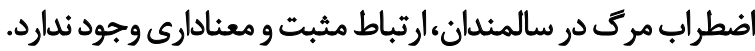
تاثير درمانهاي روانشيناختّى (معنويتدومانى و درمانهاي رفتارى) بر كاهش اضطراب مركّ در سالمثدان (معندان

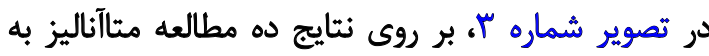

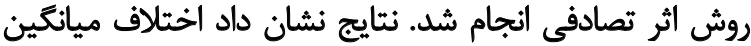

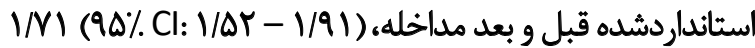

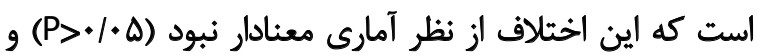

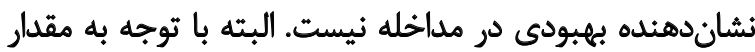

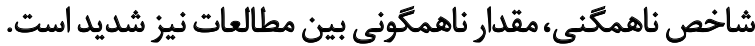

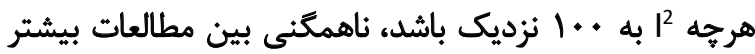

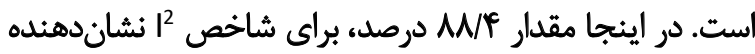

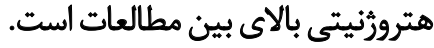

ثب

يس از اعمال معيارهاى ورود و خروج بس مقاله به مطالعه

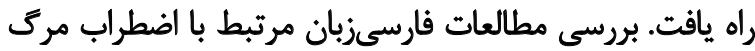

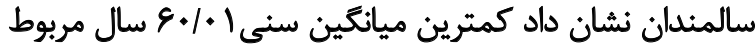

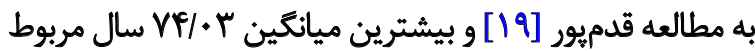

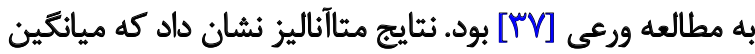

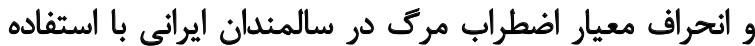

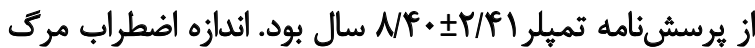

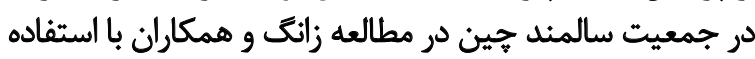

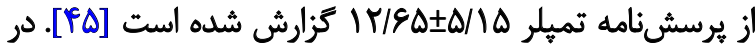

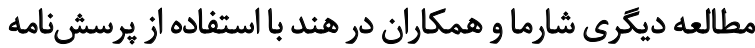

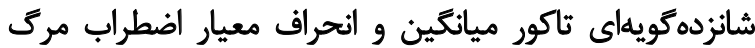
سالمندان را رانز

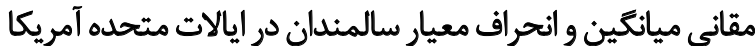

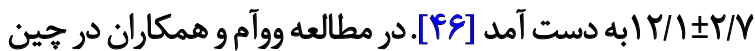

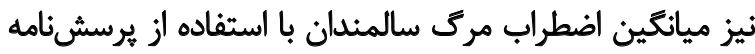

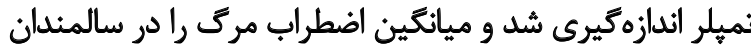

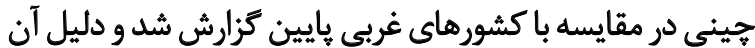

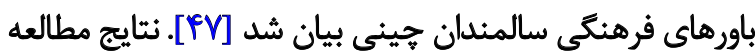

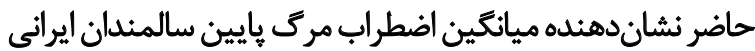

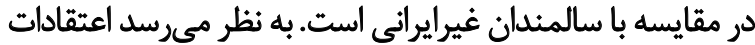

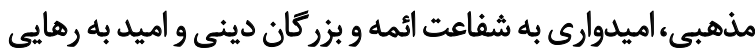

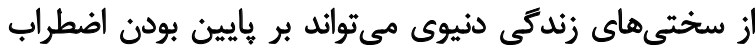

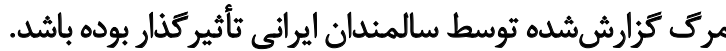
نتايج مطالعه حاضر نشان داد نمره اضطراب مرك در زنان

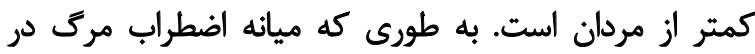

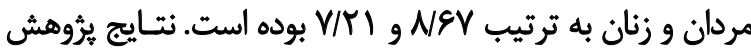




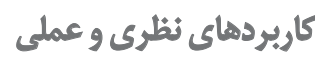

مطالعه حاضر بارويكردمرور سيستماتيك مطالعات منتشرشده

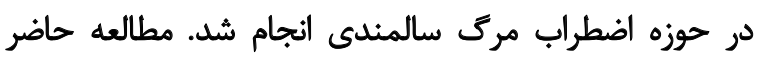

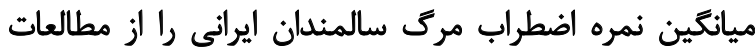

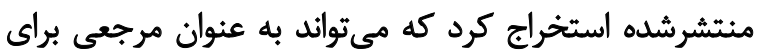

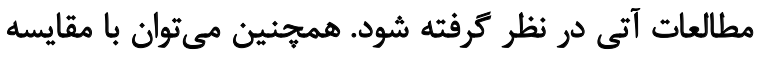

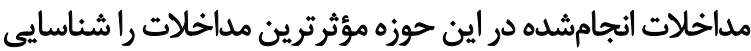

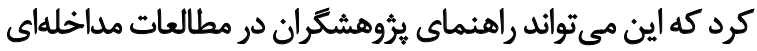

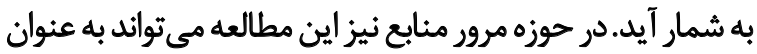

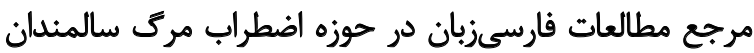

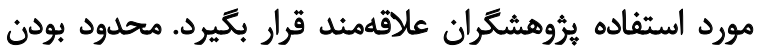

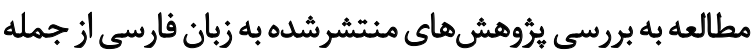

محدوديتهاى روششناختى مطالعه حاضر به شمار محرود.

ملاحظات اخلاقى

\section{ييروى أز اصول اخلاق يؤهش}

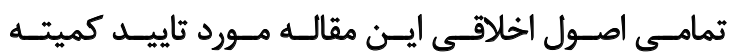

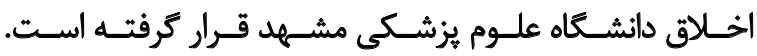

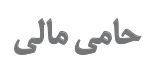

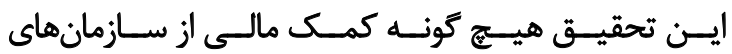

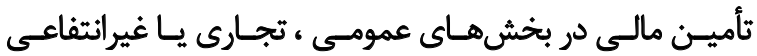

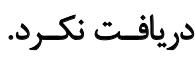

$$
\text { مشاركت ثويسند مكان }
$$

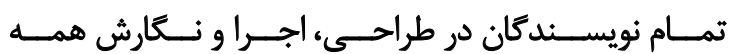

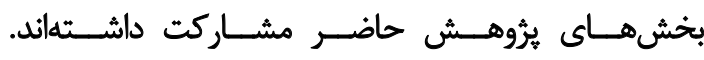

$$
\text { تعارض مناقع }
$$

بنابر اظهار نويسندكان اين مقاله تعارض منافع ندارد.
مرك در جمعيت سالمند مىشود. اين نتايج توسط مطالعات

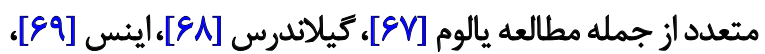

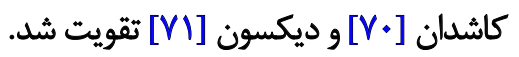

در اين يروهش سنجش هتروزنى نشان داد كه در مطالعات

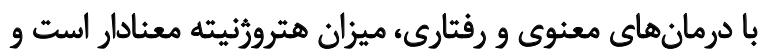

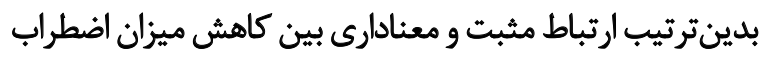

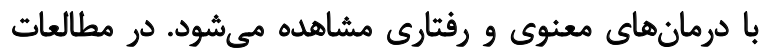

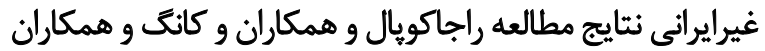

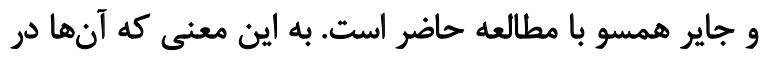

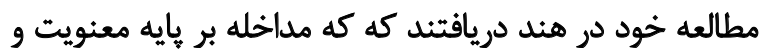

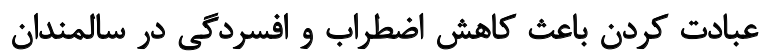

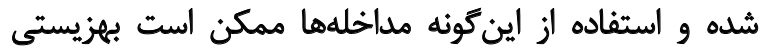

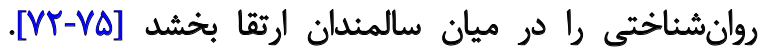

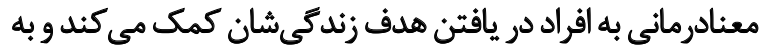

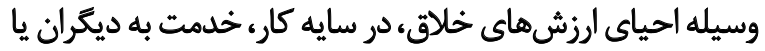

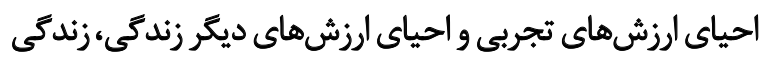

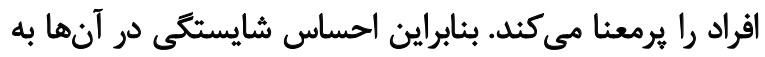

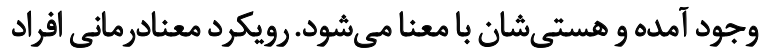

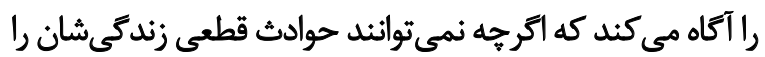

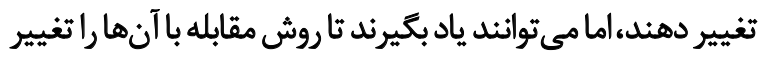

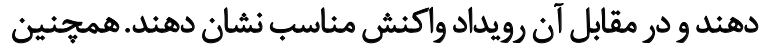

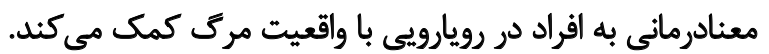

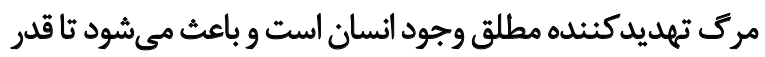

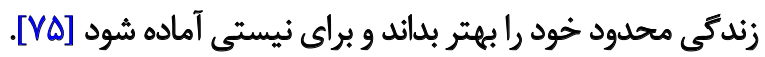

\section{تثيجهُ ئرى نهايى}

ابزارهاى متفاوت ميزان اضطراب مركى سالمندان را متفاوت

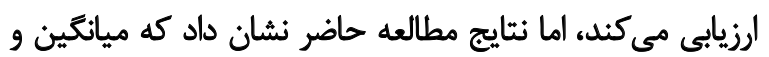

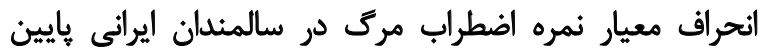

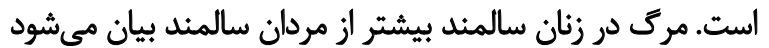

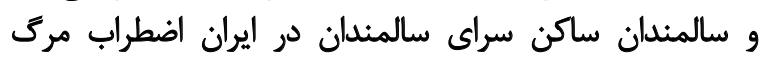

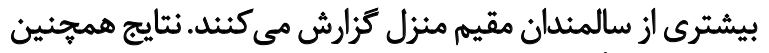

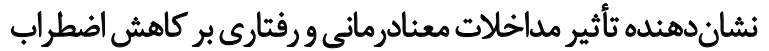

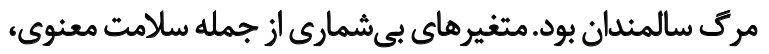

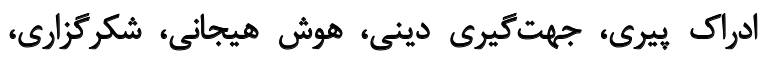
جهت

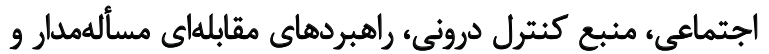

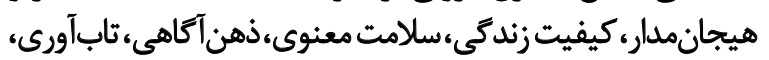

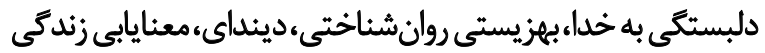

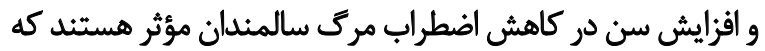

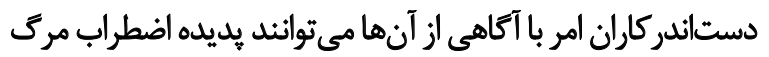

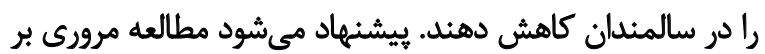

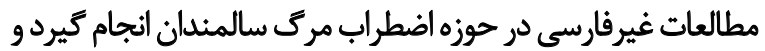
نتايج آن با مطالعه حاضر مقايسه شوده. 


\section{Refrences}

[1] United Nations, Department of Economic and Social Affairs, Population Division. World population ageing 2013 [Internet]. 2013 [Updated 2013]. Available from: https://www.un.org/ en/development/desa/population/publications/pdf/ageing/ WorldPopulationAgeing2013.pdf

[2] Salehi F, Mohsenzade F, Arefi M. [Prevalence of death anxiety in patients with breast cancer in Kermanshah, 2015 (Persian)]. Iranian Journal of Breast Diseases. 2016; 8(4):34-40. http://ijbd.ir/ article-1-487-en.html

[3] Templer DI. The construction and validation of a death anxiety scale. The Journal of General Psychology. 1970; 82(2):165-77. [DOI :10.1080/00221309.1970.9920634]

[4] Lehto RH, Stein KF. Death anxiety: An analysis of an evolving concept. Research and Theory for Nursing Practice. 2009; 23(1):2341. [DOI:10.1891/1541-6577.23.1.23]

[5] Belsky J. Developmental origins of attachment styles. Attachment \& Human Development. 2002; 4(2):166-70. [DOI:10.1080/14616730210157510]

[6] Thiemann P, Quince T, Benson J, Wood D, Barclay S. Medical students' death anxiety: Severity and association with psychological health and attitudes toward palliative care. Journal of Pain and Symptom Management. 2015; 50(3):335-42.E2. [DOI:10.1016/j. jpainsymman.2015.03.014]

[7] Caplan H. Psychiatry [M. Sadeghi, M. Sadeghi, S. Mohsenifar, V. Shariat, A. Farhoodian, V. Sharifi, Persian Trans]. Tehran: Baraye Farda Press; 2002. http://opac.nlai.ir/opac-prod/bibliographic/ 650200

[8] Brody CM, Semel VG. Strategies for therapy with the elderly: Living with hope and meaning. New York: Springer Publishing Company; 2005. https://books.google.com/ books?id=5V1mRUno0FgC\&dq

[9] Shafaii M, Payami M, Amini K, Pahlevan S. [The relationship between death anxiety and quality of life in hemodialysis patients (Persian)]. Hayat Journal. 2017; 22(4):325-38. http://hayat.tums. ac.ir/article-1-1632-en.html

[10] Yoo YS, Cho OH, Cha KS, Boo YJ. Factors influencing post-traumatic stress in Korean forensic science investigators. Asian Nursing Research. 2013; 7(3):136-41. [DOI:10.1016/j.anr.2013.07.002]

[11] Valikhani A, Firouzabadi A. [Examining death anxiety within the framework of attachment styles in cancer patients: A comparative study (Persian)]. Quarterly Journal of Health Psychology. 2016; 5(18):119-40. http://hpi.journals.pnu.ac.ir/article_3367. $\mathrm{html}$

[12] Geurtsen L. A multidimensional approach of death anxiety: Physical health, gender and psychosocial correlates in a community sample and a clinical sample of Dutch elderly people [MA. thesis]. Utrecht: Utrecht University Repository; 2010. https:// dspace.library.uu.nl/handle/1874/178776

[13] Khaki S, Khesali Z, Farajzadeh M, Dalvand S, Moslemi B, Ghanei Gheshlagh R. [The relationship of depression and death anxiety to the quality of life among the elderly population (Persian)]. Hayat Journal. 2017; 23(2):152-61. http:/ / hayat.tums.ac.ir/article1-1855-en.html

[14] Khodabakhshi-Koolaee A, Heidari F, Zangeneh Motlagh F. The effect of mixed prayer and positive psychology group therapy on life meaning and death anxiety among elderly women with physical-motor disabilities. Journal of Pizhūhish dar Dīn va Salāmat. 2018; 4(2):16-28. https://journals.sbmu.ac.ir/en-jrrh/article/ view/20681
[15] Varaee P, Momeni KM, Moradi A. Prediction of psychological well-being based on attitude toward religion and death anxiety in the elderly men of Kermanshah's part-time elderly care center. Journal of Pizhūhish dar Dīn va Salāmat. 2018; 4(2):54-68. https:/ / journals.sbmu.ac.ir/en-jrrh/article/view/20687/0

[16] Majidi A, Moradi O. [Effect of teaching the components of spiritual intelligence on death anxiety in the elderly (Persian)]. Salmand: Iranian Journal of Ageing. 2018; 13(1):110-23. [DOI:10.21859/sija.13.1.110]

[17] Bitarafan L, Kazemi M, Yousefi Afrashte M. [Relationship between styles of attachment to god and death anxiety resilience in the elderly (Persian)]. Salmand: Iranian Journal of Ageing. 2018 12(4):446-57. [DOI:10.21859/sija.12.4.446]

[18] Samadifard HR, Narimani M. [Prediction of death anxiety of elderly based on mindfulness and irrational beliefs (Persian)]. Iranian Journal of Psychiatric Nursing. 2017; 5(3):15-21. [DOI:10.21859/ijpn-05033]

[19] Ghadampour E, Moshrefi Sh. [Relationship between spiritual health, mental well-being and quality of life with death anxiety in the elderly (Persian)]. Aging Psychology. 2017; 3(2):97-106. https://jap.razi.ac.ir/article_746.html

[20] Hajiazizi AH, Bahmani B, Mahdi N, Manzari Tavakoli V, Barshan A. [Effectiveness of group logotherapy on death anxiety and life expectancy of the elderly living in boarding houses in Kerman (Persian)]. Salmand: Iranian Journal of Ageing. 2017; 12(2):220-31 [DOI:10.21859/sija-1202220]

[21] Hashemi Razini H, Baheshmat Juybari Sh, Ramshini M. [Relationship between coping strategies and locus of control with the anxiety of death in old people (Persian)]. Salmand: Iranian Journal of Ageing. 2017; 12(2):232-41. [DOI:10.21859/sija-1202232]

[22] Aqajani S, Samadifard H. [The role of cognitive fusion, locus of control and cognitive avoidance in the prediction of death anxiety in the elderly (Persian)]. Journal of Health and Care. 2017 19(1):62-74. http:// hcjournal.arums.ac.ir/article-1-705-en.html

[23] Mehri Nejad SA, Ramezan Saatchi L, Paydar S. [Death anxiety and its relationship with social support and adherence to religion in the elderly (Persian)]. Salmand: Iranian Journal of Ageing 2017; 11(4):494-503. [DOI:10.21859/sija-1104494]

[24] Golmohammadian M, Nazari H, Parvaneh A. [Effectiveness of group logo therapy on death anxiety and social adjustmen of elderly men (Persian)]. Aging Psychology. 2016; 2(3):175-67. https://jap.razi.ac.ir/article_615_en.html

[25] Dashtbozorgi Z, Sevari K, Safarzadeh S. [Effectiveness of Islamic spiritual therapy on the feeling of loneliness and death anxiety in elderly people (Persian)]. Aging Psychology. 2016; 2(3):177-86. https://jap.razi.ac.ir/article_616.html

[26] Moradi A, Shariatmadari A. [The comparison between death anxiety and loneliness among the elderly with optimistic and pessimistic life orientation (Persian)]. Aging Psychology. 2017 2(2):133-41. https://jap.razi.ac.ir/article_581.html

[27] Gholmi M, Hafezi F, Askari P, Naderi F. [The comparison between the effectiveness of mindfulness and spiritual/religious coping skills on death anxiety and blood pressure of the elderly patients with hypertension (Persian)]. Aging Psychology. 2017 2(2):143-51. https://jap.razi.ac.ir/article_580.html

[28] Zeraati M, Haghani Zemeidani M, Khodadadi Sangdeh J. [The comparison of depression and death anxiety among nursing home resident and non-resident elderlies (Persian)]. Iran Journal of Nursing. 2016; 29(102):45-54. [DOI:10.29252/ijn.29.102.45] 
[29] Sadri Demichi E, Ramezani Sh. [Effectiveness of existential therapy on loneliness and death anxiety in the elderly (Persian)]. Aging Psychology. 2016; 2(1):1-12. https://jap.razi.ac.ir/article_343.html

[30] Kakabaraei K, Moazinejad M. [The relationship between finding meaning in life and demographic characteristics with death anxiety in the elderly (Persian)]. Aging Psychology. 2016; 2(1):3747. https://jap.razi.ac.ir/article_500_en.html

[31] Dehghan K, Shariatmadar A, Kalantar Hormozi A. [Effectiveness of life review therapy on death anxiety and life satisfaction of old women of Tehran (Persian)]. Counseling Culture and Psycotherapy. 2015; 6(22):15-39. [DOI:10.22054/QCCPC.2015.4207]

[32] Moetamedi A, Pajouhinia Sh, Fatemi Ardestani SMH. [The impact of spiritual wellbeing and resiliency in predicting death anxiety among elderly people in Tehran (Persian)]. Shefaye Khatam. 2015; 3(2):19-26. [DOI:10.18869/acadpub.shefa.3.2.19]

[33] Valaei N, Zalipoor S. [The effectiveness of logo therapy on death anxiety in the elderly (Persian)]. Aging Psychology. 2015; 1(1):49-55. https:/ /jap.razi.ac.ir/article_289.html

[34] Ghorbanalipur M, Esmaeili A. [Determining the efficacy of logo therapy in death anxiety among the older adults (Persian)]. Counseling Culture and Psycotherapy. 2012;3(9):53-68. [DOI:10.22054/ QCCPC.2012.6063]

[35] Roushani Kh, Naderi F. [Spiritual intelligence, social intelligence and death anxiety associations in ahwas metropolitan senile (Persian)]. Journal of Health Psychology. 2011; 1(2):115-29. https://www.sid.ir/fa/journal/ViewPaper.aspx?ID=212565

[36] Ali Akbari Dehkordi M, Oraki M, Barghi Irani Z. [Relation betweereligious orientation with anxiety about death, and alienation in aged peoples (seniors) in Tehran (Persian)]. Quarterly Social Psychology Research. 2011; 1(2):140-59. https:/ / www.socialpsychology.ir/article_123192.html

[37] Varaee P, Momeni K, Moradi A. [Structural equation modeling: A study on the effect of religious orientation on the psychological wellbeing concerning the mediating role of death anxiety and selfcompassion in the male elderly living in Kermanshah City in 2017 (Persian)]. Salmand: Iranian Journal of Ageing. 2019; 14(2):162-77. Http://Salmandj.uswr.ac.ir/Article-1-1530-En.html

[38] Hosseini RS, Ebrahimi ME, Khalkhali V, Zamani N. [The relationship between the fear of death and religious beliefs and mental disorders in the elderly living in Karaj nursing homes (2014) (Persian)]. Salmand: Iranian Journal of Ageing. 2019; 14(2):144-61. [DOI:10.32598/sija.13.10.200]

[39] Poordad S, Momeni KM, Karami J. [Death anxiety and its relationship with social support and gratitude in older adults (Persian)]. Salmand: Iranian Journal of Ageing. 2019; 14(1):26-39. [DOI:10.32598/sija.13.10.320]

[40] Nouhi E, Karimi T, Iranmanesh S. [Comparing fear of death of the elderly settled in elderly's home and inhabited in city houses of Isfahan (Persian)]. Salmand: Iranian Journal of Ageing. 2014; 8(4):24-31. http:/ / salmandj.uswr.ac.ir/article-1-543-en.html

[41] Etemad J, Dehghan Manshadi Z, Mozafari N. [Investigation on psychometric properties of Existential Anxiety Questionnaire in elderly (Persian)]. Journal of Gerontology. 2017; 1(3):39-48. [DOI:10.18869/acadpub.joge.1.3.39]

[42] Khodabakhsh Pirkalani R, Ramezan Saatchi L, Maleki Majd M. [The effectiveness of Mindfulness-Based Cognitive Therapy (MBCT) on depression, death anxiety and life expectancy in elderly women (Persian)]. Journal of Gerontology. 2018; 3(3):11-20. [DOI:10.29252/joge.3.2.11]
[43] Salaripour SM, Maktabi GH, Alipour S. [Relationship between emotional intelligence and religious orientation with death anxiety in retired male employees of Khuzestan Steel Industries of Ahvaz (Persian)]. Counseling Culture and Psycotherapy. 2018; 9(33):99-117. [DOI:10.22054/QCCPC.2018.27614.1686]

[44] Veiskarami HA, Barzeghar Bafrooeii M, Yousefvand L. [The relationship between religious orientation and death anxiety in the elderly: The mediation role of aging perception and spiritual well-being (Persian)]. Aging Psychology. 2018; 3(4):281-91. https://jap.razi.ac.ir/article_846.html

[45] Zhang J, Peng J, Gao P, Huang H, Cao Y, Zheng L, et al. Relationship between meaning in life and death anxiety in the elderly: Self-esteem as a mediator. BMC Geriatrics. 2019; 19:308. [DOI:10.1186/s12877-019-1316-7]

[46] Assari Sh, Moghani Lankarani M. Race and gender differences in correlates of death anxiety among elderly in the United States. Iranian Journal of Psychiatry and Behavioral Sciences. 2016; 10(2):e2024. [DOI:10.17795/ijpbs-2024]

[47] Wu AMS, Tang CSK, Kwok TCY. Death anxiety among Chinese elderly people in Hong Kong. Journal of Aging and Health. 2002; 14(1):42-56. [DOI:10.1177/089826430201400103]

[48] Schumaker JF, Warren WG, Groth-Marnat G. Death anxiety in Japan and Australia. The Journal of Social Psychology. 1991; 131(4):511-8. [DOI:10.1080/00224545.1991.9713881]

[49] Cicirelli VG. Personality and demographic factors in older adults' fear of death. The Gerontologist. 1999; 39(5):569-79. [DOI:10.1093/geront/39.5.569]

[50] Depaola SJ, Griffin M, Young JR, Neimeyer RA. Death anxiety and attitudes towardthe elderly among older adults: The role of gender and ethnicity. Death Studies. 2003; 27(4):335-54. [DOI:10.1080/07481180302904]

[51] Abdel-Khalek AM. Love of life and death distress: Two separate factors. OMEGA - Journal of Death and Dying. 2007; 55(4):267-78. [DOI:10.2190/OM.55.4.b]

[52] Suhail K, Akram S. Correlates of death anxiety in Pakistan. Death Studies. 2002; 26(1):39-50. [DOI:10.1080/07481180210146]

[53] Azaiza F, Ron P, Shoham M, Gigini I. Death and dying anxiety among elderly Arab Muslims in Israel. Death Studies. 2010; 34(4):351-64. [DOI:10.1080/07481181003613941]

[54] Sharma P, Asthana HS, Gambhir IS, Ranjan JK. Death anxiety among elderly people: Role of gender, spirituality and mental health. Indian Journal of Gerontology. 2019;33(3):240-54. https:/ / www.researchgate.net/publication/337604964

[55] Fortner BV, Neimeyer RA. Death anxiety in older adults: A quantitative review. Death Studies. 1999; 23(5):387-411. [DOI:10.1080/074811899200920]

[56] Comstock GW, Partridge KB. Church attendance and health. Journal of Chronic Diseases. 1972; 25(12):665-72. [DOI:10.1016/0021-9681(72)90002-1]

[57] Busari AO. Relationship between emotional intelligence, selfesteem, anxiety and academic stress of the gifted childrenin in Oyo State, Nigeria. Global Journal of Human-Social Science. 2016; 16(5):22-34. https://globaljournals.org/GJHSS_Volume16/4Relationship-between-Emotional.pdf

[58] Maritza Espinoza V, Olivia Sanhueza A. [Fear of death and its relationship with emotional intelligence of nursing students in Concepción (Spanish)]. Acta Paulista de Enfermagem. 2012; 25(4):607-13. [DOI:10.1590/S0103-21002012000400020] 
[59] Wnuk M, Marcinkowski JT. Do existential variables mediate between religious-spiritual facets of functionality and psychological wellbeing. Journal of Religion and Health. 2014; 53(1):56-67. [DOI:10.1007/s10943-012-9597-6]

[60] Lau RWL, Cheng ST. Gratitude lessens death anxiety. European Journal of Ageing. 2011; 8(3):169. [DOI:10.1007/s10433-0110195-3]

[61] Tajvar M, Fletcher A, Grundy E, Arab M. Social support and health of older people in Middle Eastern countries: A systematic review. Australasian Journal on Ageing. 2013; 32(2):71-8. [DOI:10.1111/j.1741-6612.2012.00639.x]

[62] Khawar M, Aslam N, Aamir S. Perceived social support and death anxiety among patients with chronic diseases. Pakistan Journal of Medical Research. 2013; 52(3):75-9. https://applications.emro.who.int/imemrf/Pak_J_Med_Res/Pak_J_Med_ Res_2013_52_3_75_79.pdf

[63] Wu AMS, Tang CS, Yogo M. Death anxiety, altruism, selfefficacy, and organ donation intention among Japanese college students: A moderated mediation analysis. Australian Journal of Psychology. 2013; 65(2):115-23. [DOI:10.1111/ajpy.12003]

[64] Jans-Beken L, Lataster J, Peels D, Lechner L, Jacobs N. Gratitude, psychopathology and subjective well-being: Results from a 7.5-month prospective general population study. Journal of Happiness Studies. 2018; 19(6):1673-89. [DOI:10.1007/s10902-0179893-7]

[65] Davis DE, Choe E, Meyers J, Wade N, Varjas K, Gifford A, et al. Thankful for the little things: A meta-analysis of gratitude interventions. Journal of Counseling Psychology. 2016; 63(1):20-31. [DOI:10.1037/cou0000107]

[66] Ramírez E, Ortega AR, Chamorro A, Colmenero JM. A program of positive intervention in the elderly: Memories, gratitude and forgiveness. Aging \& Mental Health. 2014; 18(4):463-70. [DOI :10.1080/13607863.2013.856858]

[67] Yalom ID. Existential psychotherapy [S. Habib, Persian trans]. Tehran: Nashr-e Ney; 2013. http://opac.nlai.ir/opac-prod/bibliographic/3275341

[68] Gillanders DT, Sinclair AK, MacLean M, Jardine K. Illness cognitions, cognitive fusion, avoidance and self-compassion as predictors of distress and quality of life in a heterogeneous sample of adults, after cancer. Journal of Contextual Behavioral Science. 2015; 4(4):300-11. [DOI:10.1016/j.jcbs.2015.07.003]

[69] Trindade IA, Ferreira C. The impact of body image-related cognitive fusion on eating psychopathology. Eating Behaviors. 2014; 15(1):72-5. [DOI:10.1016/j.eatbeh.2013.10.014]

[70] Kashdan TB, Breen WE, Afram A, Terhar D. Experiential avoidance in idiographic, autobiographical memories: Construct validity and links to social anxiety, depressive, and anger symptoms. Journal of Anxiety Disorders. 2010; 24(5):528-34. [DOI:10.1016/j. janxdis.2010.03.010]

[71] Dickson KS, Ciesla JA, Reilly LC. Rumination, worry, cognitive avoidance, and behavioral avoidance: Examination of temporal effects. Behavior Therapy. 2012; 43(3):629-40. [DOI:10.1016/j. beth.2011.11.002

[72] Rajagopal D, Mackenzie E, Bailey Ch, Lavizzo-Mourey R. The effectiveness of a spiritually-based intervention to alleviate subsyndromal anxiety and minor depression among older adults. Journal of Religion and Health. 2002; 41(2):153-66. [DOI:10.1023/A:1015854226937]
[73] Gire JT. How death imitates life: Cultural influences on conceptions of death and dying. Online Readings in Psychology and Culture. 2002; 3(2):2. https://doi.org/10.9707/2307-0919.1120

[74] Kang KA, Im JI, Kim HS, Kim SJ, Song MK, Sim SY. The effect of logotherapy on the suffering, finding meaning, and suffering, well-being of adolescents with terminal cancer. Child Health Nursing Research. 2009; 15(2):136-44. [DOI:10.4094/ jkachn.2009.15.2.136]

[75] Kastenbaum R. Death anxiety. In: Fink G, editor. Encyclopedia of Stress. New York: Academic Press; 2007. pp. 717-722 [DOI:10.1016/B978-012373947-6.00113-6] 
This Page Intentionally Left Blank 